\title{
Integral equation theory for nematic fluids
}

\author{
M.F. Holovko \\ Institute for Condensed Matter Physics of the National Academy of Sciences of Ukraine, \\ 1 Svientsitskii Str., 79011 Lviv, Ukraine
}

Received August 13, 2010

\begin{abstract}
The traditional formalism in liquid state theory based on the calculation of the pair distribution function is generalized and reviewed for nematic fluids. The considered approach is based on the solution of orientationally inhomogeneous Ornstein-Zernike equation in combination with the Triezenberg-Zwanzig-Lovett-Mou-BuffWertheim equation. It is shown that such an approach correctly describes the behavior of correlation functions of anisotropic fluids connected with the presence of Goldstone modes in the ordered phase in the zero-field limit. We focus on the discussions of analytical results obtained in collaboration with T.G. Sokolovska in the framework of the mean spherical approximation for Maier-Saupe nematogenic model. The phase behavior of this model is presented. It is found that in the nematic state the harmonics of the pair distribution function connected with the correlations of the director transverse fluctuations become long-range in the zero-field limit. It is shown that such a behavior of distribution function of nematic fluid leads to dipole-like and quadrupole-like long-range asymptotes for effective interaction between colloids solved in nematic fluids, predicted before by phenomenological theories.
\end{abstract}

Key words: pair distribution function, integral equation theory, Maier-Saupe nematogenic model, Goldstone modes, colloid-nematic mixture

PACS: $05.20 . J j$, 05.70.Np, 61.20.-p, 68.03.-g

\section{Introduction}

The pair distribution function $g(12)$ plays central role in the modern fluid theory. It establishes a bridge between microscopic properties modeled by interparticle interactions and the macroscopic ones such as structural, thermodynamic, dielectric and other properties. For homogeneous fluids the integral equation methods have been intensively used in fluid theory during the last decades [1, 2]. This technique is based on the analytical or numerical calculation of the pair distribution function by the solution of the Ornestein-Zernike (OZ) equation within different closures: PercusYevick (PY), hypernetted chain (HNC), mean spherical approximation (MSA) and its different modifications. In the presence of an external field the fluid becomes inhomogeneous and is described by the singlet distribution function $\rho(1)$ that appears instead of the bulk density of the homogeneous liquid [3]. The external field determines the symmetry of the singlet distribution function and its dependence on coordinates of the fixed molecule 1. The transition from homogeneous to inhomogeneous state leads to the broken symmetry of the system. As a result, the pair distribution function of inhomogeneous fluids loses the uniform invariance and does not have the symmetry of the pair potential. Besides external fields, the inhomogeneity can also be caused by a change of the system symmetry as a result of phase transition. Such a typical situation takes place in the case of crystallization, where at certain values of the density the periodic singlet distribution function branches off the uniform one.

Thus, in the inhomogeneous case, the $\mathrm{OZ}$ equation includes the singlet distribution function $\rho(1)$ and, besides the closure for the $\mathrm{OZ}$ equation, an additional relation between singlet and pair distribution functions is needed [1-3]. There are at least two exact relations that can be used for this aim. It could be the first member from the hierarchy of the Bogolubov-Born-GreenKirkwood-Yvon (BBGKY) equation [1, 4] or the Triezenberg-Zwanzig-Lovett-Mou-Buff-Wertheim (TZLMBW) equation [1, 5 7]. We should note that in accordance with Bogolubov's idea about the 
quasiaverages [8] for a correct treatment of the system with spontaneously broken symmetries, an external field of infinitely small value should be introduced in order to stabilize the system.

For molecular fluids, the inhomogeneity can be caused by the broken rotational invariance in addition to the break of the translational invariance. Such a situation appears at the phase transition from isotropic to nematic liquid crystal phase, when at certain thermodynamical conditions the orientational-dependent singlet distribution function branches off the isotropic one. Similarly to the crystallization case, the pair distribution function loses the translational invariant form as a result of the broken symmetry. It loses its rotationally invariant form in the nematic case. The change of the fluid from isotropic to nematic state in the absence of external fields induces collective fluctuations, which develops orientational wave excitations, the so-called Goldstone modes. This leads to the divergence of the corresponding harmonics of the pair distribution function in the limit of zero wave vector $k$.

Recently much effort has been devoted to generalization of the integral equation theory to orientationally ordered (anisotropic) fluids. In order to investigate the properties of molecular fluids in the nematic phase some Ansatzes based on the construction of an effective isotropic state are used [9, 10]. Another description of the isotropic-nematic phase transition is connected with the application of the TZLMBW equation, with the assumption that the direct correlation function in the nematic phase can be approximated to it by the form which is reduced in the isotropic case, i.e., by its rotationally invariant form. This procedure was used by Lipszyc and Kloczkowski [11] and Zhong and Petschek [12, 13]. They made an attempt to calculate the single-particle distribution function and the pair distribution functions in a self-consistent way on the basis of the OZ equation and the so-called Ward identity. The Ward identity relates the singlet distribution function to an integral of the pair direct correlation function. Later Holovko and Sokolovska [14] showed that this is nothing else than the TZLMBW equation in the functional differential form. Treating the direct correlation function in the PY approximation as the effective potential, Zhong and Petschek [12, 13] supposed that the direct correlation function should be rotationally invariant just like the initial potential. In order to remake the PY closure in a rotationally invariant form, they used a procedure named in [15] as an unoriented nematic approximation. It was shown that with the modified PY closure, the Ward identity is implemented and yields an infinite susceptibility in the limit of zero wave vector for the Goldstone modes. However, Holovko and Sokolovska [14] showed that the requirement of the rotational invariance for correlation functions leads to an incorrect conclusion about the divergence of the nematic structure factor in the limit of zero wave vector. In contrast to the TZLMBW, the BBGKY equation does not reproduce the correct zero-field divergence in the transverse susceptibility of nematic fluids [16]. Thus, it seems better to build a theory using the TZLMBW equation instead of the BBGKY one.

The generalization of the integral equation theory for orientationally inhomogeneous molecular fluids was formulated by Holovko and Sokolovska [14, 17]. In this approach the self-consistent solution of the OZ and TZLMBW equations are used for the calculation of the pair and singleparticle distribution functions in nematics. The developed method does not impose any additional approximations other than a closure for the OZ equation. A principal point of this approach is the use of exact relations obtained from TZLMBW equation for the nematic phase. In accordance with the Bogolubov idea [8] there was introduced an external field of infinitely small value which fixes the orientation of the nematic director. It was shown that the application of TZLMBW equation provides a correct description of the Goldstone modes in full accordance with the fluctuation theory of de Gennes [18]. Only harmonics of the distribution function connected with correlations of the director transverse fluctuations have to diverge at $\kappa=0$, the others being finite.

The developed integral equation approach was applied to the hard sphere Maier-Sauper nematic model. There was obtained an analytical solution for this model in MSA approximation [14, 19], which was used for the description of phase behaviour of the considered model [20]. The properties of hard sphere Maier-Saupe model were also studied by numerical solution of orientationally inhomogeneous OZ equation in PY, MSA, HNC and reference HNC approximations [16, 21, 22]. The considered integral equation theory was also applied to the investigation of a planar nematic fluid in the presence of a disoriented field [23 26]. The obtained analytical results in MSA approximation 
for hard sphere Maier-Saupe nematic model were used in Henderson-Abraham-Barker approach 1, 27 for the investigation of a nematic fluid near hard wall in the presence of orienting field [28, 29]. These results were applied to the investigations of colloidal interactions in nematic-colloid dispersions 30 35].

This paper reviews the recent studies of nematic fluids within the framework of integral equation theory for orientationally inhomogeneous molecular fluids. This review is devoted to the memory of Tatjana Sokolovska who passed away a year ago. The remainder of the paper is organized as follows. The general formulation of integral equation, the orientational expansions of the pair correlation and the singlet distribution functions are presented in the first section. The solution of the MSA for hard sphere Maier-Saupe nematic model in the presense and in the absence of an external field is discussed in the second section. In that section, thermodynamic properties and phase behavior of this model are discussed. In the third section the integral equation approach is used for the description of a nematic fluid near hard wall that interacts with a uniform orienting field. Some aspects of intercolloidal interactions in a nematic fluid are studied by integral equation theory and are also discussed in this section.

\section{Integral equations for orientationally inhomogeneous fluids: general relations}

In this paper we consider a fluid of spherical particles with diameter $\sigma$ having an orientation specified by the unit vector $\boldsymbol{\omega}$. The fluid is subject to an external ordering field of the form

$$
v(1)=-W_{2} \sqrt{5} P_{2}\left(\cos \vartheta_{1}\right) \text { with } W_{2} \geqslant 0
$$

which favors an order parallel to the direction $\boldsymbol{n}, P_{2}(\cos \vartheta)=\frac{3}{2}\left(\cos ^{2} \vartheta-1\right)$ - is a Legendre polynomial of second order, $\vartheta$ is the angle between vectors $\boldsymbol{\omega}$ and $\boldsymbol{n}, 1$ indicates both position $r_{1}$ and orientation $\omega_{1}$ of the molecule.

We confine that in the interactions between the fluid particles, the orientational component is essentially a Maier-Saupe term [36] and assume that the intermolecular potential $v(12)$ can be presented in the form

$$
v(1,2)=v_{h}\left(r_{12}\right)+v_{0}\left(r_{12}\right)+v_{2}\left(r_{12}, \omega_{1}, \omega_{2}\right)
$$

where $v_{h}\left(r_{12}\right)$ is the hard sphere potential

$$
v_{h}(r)= \begin{cases}\infty, & \text { for } \quad r<\sigma \\ 0, & \text { for } r>\sigma\end{cases}
$$

The long-range attraction has an isotropic part

$$
v_{0}(r)=-A_{0} \frac{\exp \left(-z_{0} r\right)}{r}
$$

and an anisotropic part

$$
v_{2}\left(r, \omega_{1}, \omega_{2}\right)=-A_{2} \frac{\exp \left(-z_{2} r\right)}{r} P_{2}\left(\cos \vartheta_{12}\right)
$$

where $P_{2}\left(\cos \vartheta_{12}\right)$ is the second Legendre polynomial, $\vartheta_{12}$ is the angle between the axes of molecules 1 and 2 . The parameters $z_{0}, z_{2}$ and $A_{0}, A_{2}$ determine the range and the strength of the coupling interactions.

The molecular Ornstein-Zernike equation for orientational inhomogeneous fluids can be written in the form [1-3]

$$
h(1,2)=C(1,2)+\int \mathrm{d} 3 \rho(3) C(1,3) h(3,2)
$$

where $\mathrm{d} 3=\mathrm{d} r_{3} \mathrm{~d} \omega_{3}, h(12)=g(12)-1$ and $C(12)$ are, respectively, the total and direct correlation functions. 
A some closure relation which relates the correlation functions $C(12)$ and $h(12)$ to the pair potential $v(12)$ should be added. In this paper we will use MSA closure, according to which

$$
\begin{array}{lll}
h(1,2)=-1 & \text { for } & r_{12}<\sigma, \\
C(1,2)=-\beta v(1,2) & \text { for } & r_{12}>\sigma .
\end{array}
$$

Condition (1.7) is exact for the considered model since $g_{12}=0$ for $r_{12}<\sigma$. The condition (1.8) assumes that the long-range asymptote of $C(12)=-\beta v(12)$ is correct for the whole intermolecular distance $r>\sigma$.

For orientational inhomogeneous fluid $\rho(1)=\rho f(\omega)$, where $\rho$ is the number density of the ordered phase, $f(\omega)$ is a single particle distribution function which can be written in the form [37]

$$
f(w)=\frac{1}{Z} \exp (-\beta v(1)+C(1))
$$

where the constant $Z$ can be found from the normalization condition

$$
\int f(\omega) \mathrm{d} \omega=1,
$$

$\beta=\frac{1}{k_{\mathrm{B}} T}, k_{\mathrm{B}}$ is the Boltzmann constant, $T$ is the temperature, $C(1)$ is the singlet direct correlation function, which is the first in the hierarchy of direct correlation functions.

By using the functional differentiation technique we can define the total and the direct pair correlation functions as 37 ]

$$
\begin{aligned}
-\frac{1}{\beta} \frac{\delta \rho(1)}{\delta v(2)} & =\rho(1) \delta(1,2)+\rho(1) \rho(2) h(1,2), \\
-\beta \frac{\delta v(1)}{\delta \rho(2)} & =\frac{\delta(1,2)}{\rho(1)}-C(1,2) .
\end{aligned}
$$

where $\delta(12)$ is the Dirac $\delta$-function of all coordinates of the molecules 1 and 2 . In accordance with (1.9) the second of these relations can be written in the form of Ward identity introduced by Zhong and Petschek [12, 13]

$$
\frac{\delta C\left(\omega_{1}\right)}{\delta \rho\left(\omega_{2}\right)}=\int \mathbf{d r}_{12} C\left(r_{12}, \omega_{1}, \omega_{2}\right)
$$

It is important to note that after the inclusion of an external field $v(1)$, the system instead of a rotational invariance possesses a rotational covariance [8]. This means that the Hamiltonian and the average values, like correlation functions, are rotationally invariant if the external field and the molecules are simultaneously rotated. As a result of this symmetry, one gets

$$
\begin{aligned}
& \nabla_{\omega_{1}} \rho\left(\omega_{1}\right)=\int \mathrm{d} r_{12} \mathrm{~d} \omega_{2} \frac{\delta \rho\left(\omega_{1}\right)}{\delta v\left(\omega_{2}\right)} \nabla_{\omega_{2}} v\left(\omega_{2}\right), \\
& \nabla_{\omega_{1}} v\left(\omega_{1}\right)=\int \mathrm{d} r_{12} \mathrm{~d} \omega_{2} \frac{\delta v\left(\omega_{1}\right)}{\delta \rho\left(\omega_{2}\right)} \nabla_{\omega_{2}} \rho\left(\omega_{2}\right)
\end{aligned}
$$

where for the considered case of linear molecules [38]

$$
\boldsymbol{\nabla}_{\omega}=[\hat{\mathbf{r}} \times \nabla]=-\mathbf{e}_{\vartheta} \frac{1}{\sin \vartheta} \frac{\partial}{\partial \phi}+\mathbf{e}_{\phi} \frac{\partial}{\partial \vartheta}
$$

is the angular gradient operator, $e_{\omega}$ and $e_{\varphi}$ are two orthogonal unit vectors perpendicular to the unit vector $\hat{\mathbf{r}}$.

Combination of the relations (1.11)-(1.12) and (1.14)-(1.15) yields integro-differential equations for the singlet distribution function - the TZLMBW equations for spatially homogeneous but 
orientationally non-uniform systems

$$
\begin{aligned}
& \beta \nabla_{\omega_{1}} v\left(\omega_{1}\right)+\nabla_{\omega_{1}} \ln \rho\left(\omega_{1}\right)=-\beta \int \mathbf{d} \mathbf{r}_{12} \mathrm{~d} \omega_{2} h\left(r_{12}, \omega_{1}, \omega_{2}\right) \rho\left(\omega_{2}\right) \nabla_{\omega_{2}} v\left(\omega_{2}\right), \\
& \beta \nabla_{\omega_{1}} v\left(\omega_{1}\right)+\nabla_{\omega_{1}} \ln \rho\left(\omega_{1}\right)=\int \mathbf{d} \mathbf{r}_{12} \mathrm{~d} \omega_{2} C\left(r_{12}, \omega_{1}, \omega_{2}\right) \nabla_{\omega_{2}} \rho\left(\omega_{2}\right) .
\end{aligned}
$$

From equation (1.17) it follows directly that to have a non-trivial solution for $\rho\left(\omega_{1}\right)$ the integral $\int \mathbf{d r}_{12} h\left(r_{12}, \omega_{1}, \omega_{2}\right)$ should diverge in the limit $v(\omega) \rightarrow 0^{+}$. The divergence signals the appearance of the Goldstone modes.

The equation (1.18) in the zero-field limit can be written in the form

$$
\nabla_{\omega_{1}} \ln \rho\left(\omega_{1}\right)=\int C\left(\omega_{1}, \omega_{2}\right) \nabla_{\omega_{2}} \rho\left(\omega_{2}\right) \mathrm{d} \omega_{2}
$$

which is an integro-differential form of the Ward identity $(1.13), C\left(\omega_{1}, \omega_{2}\right)=\int C\left(r, \omega_{1}, \omega_{2}\right) \mathbf{d r}$.

The next step of the integral equation theory for molecular fluids is usually connected with spherical harmonics expansions for orientational dependent functions $g(12)$ or $h(12), c(12)$ and $f(\omega)$. Due to orientational inhomogeneity of the fluid the traditional orientational invariance technique [1, 38] should be slightly modified [14].

In uniaxial fluids, the orientational distribution function $f(\omega)$ is axially symmetric around a preferred direction $\mathbf{n}$ and depends only on the angle $\vartheta$ between the molecular orientation $\boldsymbol{\omega}$ and $\boldsymbol{n}$. It allows us to write the relation $(1.9)$ for $f(\omega)$ in the form

$$
f(\omega)=\frac{1}{Z} \exp \left\{\sum_{l>0} B_{l} Y_{l 0}(\omega)\right\}
$$

where the spherical harmonics $Y_{l m}(\omega)$ satisfy the standard Condon-Shortey phase convention [38].

The nematic ordering is defined by the parameters

$$
S_{l}=\left\langle P_{l}(\cos \vartheta)\right\rangle=\int \mathrm{d} \omega f(\omega) P_{l}(\cos \vartheta),
$$

where $P_{l}(\cos \vartheta)=\sqrt{\frac{1}{2 l+1}} Y_{l, 0}(\omega)$ are the Legendre polynomials.

In the space-fixed coordinate system with $z$-axis parallel to $\mathbf{n}$ the direct and total pair correlation functions can be written in the form

$$
f\left(r, \omega_{1}, \omega_{2}\right)=\sum_{\substack{m, n, l \\ \mu, \nu, \lambda}} f_{m n l}^{\mu \nu \lambda}(r) Y_{m \mu}\left(\omega_{1}\right) Y_{n \nu}^{*}\left(\omega_{2}\right) Y_{l \lambda}\left(\omega_{r}\right)
$$

where $f\left(r, \omega_{1}, \omega_{2}\right)=h(12)$ or $C(12), \mathbf{r}$ is a separation vector of molecular mass center, $\omega_{r}$ being its orientation.

Due to invariance of a uniaxial system with respect to rotations around $z$-axis, $\mu+\lambda=\nu$. Since the pair potential (1.2) is independent of orientation of the intermolecular separation vector $\mathbf{r}$, the harmonic coefficients that survive in the expansion (1.22) have only $l=\lambda=0$ and $\mu+\nu=0$. This permits to attain notational simplification from six indexes to three. In the MSA for the considered model, the expansion (1.22) reduces to

$$
f\left(r, \omega_{1}, \omega_{2}\right)=f_{000}(r)+f_{200}(r)\left[Y_{20}\left(\omega_{1}\right)+Y_{20}\left(\omega_{2}\right)\right]+\sum_{|\mu| \leq 2} f_{22 \mu}(r) Y_{2 \mu}\left(\omega_{1}\right) Y_{2 \mu}^{*}\left(\omega_{2}\right) .
$$

It should be noted that for isotropic case $f_{200}(r)=0$.

Due to the uniaxial symmetry of a nematic the OZ equations for harmonics with different values of $\mu$ decouple. In the MSA for $\mu \neq 0$ harmonics, we have

$$
h_{22 \mu}\left(r_{12}\right)=C_{22 \mu}\left(r_{12}\right)+\rho\left\langle Y_{2 \mu}^{2}(\omega)\right\rangle_{\omega} \int C_{22 \mu}\left(r_{13}\right) h_{22 \mu}\left(r_{32}\right) \mathrm{d} \mathbf{r}_{3}
$$


with the closure

$$
\begin{aligned}
h_{22 \mu}\left(r_{12}\right) & =0, & r_{12} & <\sigma, \\
C_{22 \mu}\left(r_{12}\right) & =\beta \frac{1}{5} A_{2} \frac{1}{r_{12}} \mathrm{e}^{-z_{2} r_{12},} & r_{12} & >\sigma
\end{aligned}
$$

where $\langle\ldots\rangle_{\omega}=\int f(\omega)(\ldots) \mathrm{d} \omega$. The spherical harmonics $Y_{m \mu}(\omega)$ are normalized in such a way that

$$
\int Y_{m \mu}(\omega) Y_{n \nu}^{*}(\omega) \mathrm{d} \omega=\delta_{m n} \delta_{\mu \nu} .
$$

For the case $\mu=0$, we obtained a more complex $\mathrm{OZ}$ equation. In Fourier space it may be presented in a matrix form

$$
\hat{H}(k)=\hat{C}(k)+\hat{C}(k) \hat{\rho} \hat{H}(k),
$$

where

$$
\begin{gathered}
\hat{H}(k)=\left(\begin{array}{cc}
h_{000}(k) & h_{020}(k) \\
h_{200}(k) & h_{220}(k)
\end{array}\right), \\
\hat{C}(k)=\left(\begin{array}{cc}
C_{000}(k) & C_{020}(k) \\
C_{200}(k) & C_{220}(k),
\end{array}\right), \\
\hat{\rho}=\rho\left(\begin{array}{cc}
1 & \left\langle Y_{20}(\omega)\right\rangle_{\omega} \\
\left\langle Y_{20}(\omega)\right\rangle_{\omega} & \left\langle Y_{20}^{2}(\omega)\right\rangle_{\omega}
\end{array}\right), \\
h_{m n \mu}(k)=4 \pi \int_{0}^{\infty} r^{2} \mathrm{~d} r \frac{\sin k r}{k r} h_{m n \mu}(r) .
\end{gathered}
$$

The closures of the equation (1.27) in the $r$-space are as follows for $r<\sigma$ :

$$
\begin{aligned}
& h_{000}(r)=-1, \\
& h_{020}(r)=h_{200}(r)=h_{220}(r)=0,
\end{aligned}
$$

and for $r>\sigma$ :

$$
\begin{aligned}
C_{000}(r) & =\beta A_{0} \frac{\mathrm{e}^{-z_{0} r}}{r}, \\
C_{020}(r) & =C_{200}(r)=0, \\
C_{220}(r) & =\frac{1}{5} \beta A_{2} \frac{\mathrm{e}^{-z_{2} r}}{r} .
\end{aligned}
$$

The space-fixed $x, y, z$-components of the angular gradient operator are given by $\boldsymbol{\nabla}_{\omega}=i \mathbf{l}$, where $\mathbf{l}$ is the angular momentum operator. Using the relations [38]

$$
\begin{gathered}
\left(\nabla_{\omega}\right)_{y}=\frac{l_{+}-l_{-}}{2}, \\
l_{ \pm} Y_{m \mu}(\omega)=[m(m+1)-\mu(\mu+1)]^{\frac{1}{2}} Y_{m, \mu \pm 1}(\omega)
\end{gathered}
$$

and expressions (1.20), (1.22) the y-component of (1.18) is obtained in the form

$$
\begin{gathered}
\sum_{l} \sqrt{l(l+1)}\left(B_{l}+\beta W_{2} \sqrt{30}\right)\left[Y_{l, 1}\left(\omega_{1}\right)-Y_{l,-1}\left(\omega_{1}\right)\right]=\sum_{l^{\prime}} \sum_{m n \mu} \int C_{m n 0}^{\mu \mu 0}(r) Y_{m \mu}\left(\omega_{1}\right) \\
\times Y_{n \mu}^{*}\left(\omega_{2}\right) \sqrt{l^{\prime}\left(l^{\prime}+1\right)} B_{l^{\prime}}\left[Y_{l^{\prime}, 1}\left(\omega_{2}\right)-Y_{l^{\prime}, 1}\left(\omega_{2}\right)\right] \rho\left(\omega_{2}\right) \mathrm{d} \omega_{2} \mathrm{~d} \mathbf{r}
\end{gathered}
$$


Taking into account that only quantities independent of the azimuthal angle $\varphi$ yield non-zero average values and using the orthogonality of $Y_{l m}(\omega)$ one gets the following equation

$$
\mathbf{L}=\hat{C} \hat{Y} \mathbf{L}+\beta W_{2} \sqrt{30} \mathbf{l},
$$

where $\mathbf{L}$ is a column with $L_{l}=\sqrt{l(l+1)} B_{l}, \hat{C}$ and $\hat{Y}$ are matrices with elements

$$
\begin{aligned}
C_{m n} & =\int \mathrm{d} \mathbf{r} C_{m n 0}^{110}(r), \\
Y_{m n} & =\rho \int \mathrm{d} \omega f(\omega) Y_{m 1}(\omega) Y_{n 1}^{*}(\omega),
\end{aligned}
$$

$\mathbf{l}$ is a column with $\delta_{l, 2}$. After integration by parts the equation (1.18) can be written in the form

$$
\beta \nabla_{\omega_{1}} v(1)+\nabla_{\omega_{1}} \ln \rho\left(\omega_{1}\right)=-\int \rho\left(\omega_{2}\right) \mathrm{d} \omega_{2} \nabla_{\omega_{2}} C_{2}\left(\omega_{1}, \omega_{2}\right) .
$$

Hence,

$$
\mathbf{L}=\hat{C} \mathbf{P}+\beta W_{2} \sqrt{301}
$$

where $\mathbf{P}$ is the column with

$$
P_{l}=\rho \sqrt{l(l+1)(2 l+1)} S_{l} .
$$

Equations (1.37) and (1.41) connect the system order parameters $S_{l}$, zero Fourier transforms of the direct correlation function harmonics $C_{m n 0}^{110}(r)$, the intensity of external field $W_{2}$ and the coefficients $B_{l}$ of the single particle distribution function $f(\omega)$. In the MSA approximation for the considered model $(m, n)=(0,2)$ and the equations (1.37) and (1.41) reduce to

$$
\begin{aligned}
& B_{2}=C_{22} Y_{22} B_{2}+\beta W_{2} \sqrt{5}, \\
& B_{2}=5 \rho C_{22} S_{2}+\beta W_{2} \sqrt{5} .
\end{aligned}
$$

As a result, for a single particle distribution function $f(\omega)$ we will have

$$
f(\omega)=\frac{\sqrt{\frac{3}{2} \beta W_{2}^{\text {eff }}}}{D\left(\sqrt{\frac{3}{2} \beta W_{2}^{\text {eff }}}\right)} \exp \left[\beta W_{2}^{\text {eff }} P_{2}(\cos \vartheta)\right],
$$

where

$$
\beta W_{2}^{\mathrm{eff}}=\frac{\beta W_{2} \sqrt{5}}{1-C_{22} Y_{22}}
$$

$D(x)$ is Dawson's integral

$$
D(x)=\mathrm{e}^{-x^{2}} \int_{0}^{x} \mathrm{e}^{y^{2}} \mathrm{~d} y .
$$

In the absence of the external field $W_{2}=0$ and in accordance with (1.44), (1.45)

$$
\begin{aligned}
1 & =C_{22} Y_{22}, \\
B_{2} & =5 \rho C_{22} S_{2} .
\end{aligned}
$$

Thus, in the absence of any field a the single distribution function $f(\omega)$, the problem results in the well-known Maier-Saupe equation [36]

$$
S_{2}=\frac{\int P_{2}(\cos \vartheta) \exp \left[M S_{2} P_{2}(\cos \vartheta)\right] \mathrm{d} \omega}{\int \exp \left[M S_{2} P_{2}(\cos \vartheta)\right] \mathrm{d} \omega}
$$


where

$$
M=\frac{5}{\left\langle\left|Y_{21}(\omega)\right|^{2}\right\rangle_{\omega}} .
$$

After integration the equation (1.50) can be written in the form

$$
\begin{aligned}
S_{2} & =\frac{3}{4}\left[\frac{1}{x D(x)}-\frac{1}{x^{2}}\right]-\frac{1}{2}, \\
M S_{2} & =\frac{2}{3} x^{2} .
\end{aligned}
$$

The Maier-Saupe theory predicts a first-order phase transition from isotropic phase with $S_{2}=0$ to nematic phase $S_{2} \neq 0$. From the OZ equation for $\mu \neq 0$ (1.24) using the equation (1.48) it is not difficult to prove that in the absence of external field the harmonics with $\mu= \pm 1$ have a divergence. This divergence is connected with Goldstone modes. In the MSA approach for the considered model, this is the harmonic $h_{221}(r)$.

\section{Hard sphere Maier-Saupe model: MSA description}

In this section we consider the analytical solution of $\mathrm{OZ}$ equation with MSA closure for the model considered in previous section. The obtained results will be used for the description of structure, thermodynamics, and phase behavior of this model. By the factorization method of Baxter and Wertheim [19, 39] the integral equation (1.24) for $\mu \neq 0$ under conditions (1.25) can be reduced to a system of algebraic equations

$$
\begin{aligned}
\frac{12}{5} \eta\left\langle\left|Y_{2 \mu}(\omega)\right|^{2}\right\rangle_{\omega} \frac{\beta A_{2}}{\sigma} & =D\left(1-\tilde{Q}_{2 \mu}\left(z_{2}\right)\right) \\
2 \pi \tilde{g}_{22 \mu}\left(z_{2}\right)\left[1-\tilde{Q}_{2 \mu}\left(z_{2}\right)\right] & =\frac{D}{2} \exp \left[-2 z_{2} \sigma\right]\left[1-2 \pi g_{22 \mu}\left(z_{2}\right)\right] \\
-C & =\left[1-2 \pi g_{22 \mu}\left(z_{2}\right)\right] D
\end{aligned}
$$

where $\eta=\frac{1}{6} \pi \rho \sigma^{3}, \mathrm{C}$ and $\mathrm{D}$ are dimensionless coefficients of the Baxter factor correlation function

$$
Q_{2 \mu}(r)=\frac{z}{\rho\left\langle\left|Y_{2 \mu}(\omega)\right|^{2}\right\rangle_{\omega}}\left[q_{0 \mu}(r)+D \mathrm{e}^{-z_{2} r}\right]
$$

with the short-range part

$$
q_{0 \mu}(r)=\left\{\begin{array}{lr}
C\left[\mathrm{e}^{-z_{2} r}-\mathrm{e}^{-z_{2} \sigma}\right], & r<\sigma, \\
0, & r>\sigma,
\end{array}\right.
$$

$\tilde{Q}_{2 \mu}\left(z_{2}\right)$ and $\tilde{g}_{22 \mu}\left(z_{2}\right)$ are the dimensionless Laplace transforms of $Q_{2 \mu}(r)$ and $h_{22 \mu}(r)$

$$
\begin{aligned}
\tilde{Q}\left(z_{2}\right) & =\rho\left\langle\left|Y_{21}(\omega)\right|^{2}\right\rangle_{\omega} \int_{0}^{\infty} \mathrm{e}^{-z_{2} t} Q(t) \mathrm{d} t, \\
\tilde{g}_{221}\left(z_{2}\right) & =\frac{\rho\left\langle\left|Y_{21}(\omega)\right|^{2}\right\rangle_{\omega}}{z_{2}} \int_{\sigma}^{\infty} \mathrm{e}^{-z_{2} t_{h_{221}}(t) t \mathrm{~d} t .}
\end{aligned}
$$

From the definition of the factor correlation function it follows that

$$
1-\rho\left\langle\left|Y_{2 \mu}(\omega)\right|^{2}\right\rangle_{\omega} \int C_{22 \mu}(r) \mathrm{d} \mathbf{r}=\left|Q_{2 \mu}(k=0)\right|^{2}
$$

where

$$
Q_{2 \mu}(k)=1-\rho\left\langle\left|Y_{2 \mu}(\omega)\right|^{2}\right\rangle_{\omega} \int_{0}^{\infty} \mathrm{d} r \mathrm{e}^{\mathrm{i} k r} Q(r)
$$


The joint use of (2.8) for $\mu=1$ and (1.43) gives us the additional equation to determine $\rho\left\langle\left|Y_{21}(\omega)\right|^{2}\right\rangle_{\omega}$

$$
Q_{21}(k=0)=\sqrt{\frac{\beta W_{2}}{B_{2}}}
$$

and in the explicit form

$$
f=D+d c, \quad f=1-\sqrt{\frac{\beta W_{2}}{B_{2}}}
$$

where $d=\mathrm{e}^{-z_{2} \sigma} \Delta_{1}\left(z_{2} \sigma\right)$. Here and below we use the symbols

$$
\Delta_{n}(x)=\mathrm{e}^{x}-\sum_{l=0}^{n} \frac{1}{l !} x^{l}
$$

Formulas (2.11), (2.2), and (2.3) yield the expression for D

$$
D=-\frac{1}{2 a}\left(b+\sqrt{b^{2}-4 a c}\right)
$$

where

$$
\begin{aligned}
a & =-d \exp \left(-2 z_{2} \sigma\right)-(d-1)\left[d-f \Delta_{0}^{2}\left(-z_{2} \sigma\right)\right] \\
b & =(d-1) \frac{c}{f}+f\left[\Delta_{0}^{2}\left(-z_{2} \sigma\right) f-d\right]+d f \exp \left(-z_{2} \sigma\right) \\
c & =f\left[2 d-\Delta_{0}^{2}\left(z_{2} \sigma\right)\right]
\end{aligned}
$$

Now from equation (2.1) for $\mu=1$ we can obtain the dependence between the ordering parameter $\rho\left\langle\left|Y_{21}(\omega)\right|^{2}\right\rangle_{\omega}$ and the system parameters $\eta, \beta A_{2} \frac{1}{\sigma}, \frac{W_{2} \sigma}{A_{2}}$ and $z_{2} \sigma$

$$
\frac{\beta A_{2}}{\sigma} \eta \rho\left\langle\left|Y_{21}(\omega)\right|^{2}\right\rangle_{\omega}=\frac{5}{24} D\left[2-\frac{f}{d} \Delta_{0}^{2}\left(z_{2} \sigma\right)-D\left(1-\frac{\Delta_{0}^{2}\left(-z_{2} \sigma\right)}{d}\right)\right]
$$

In the absence of external field $\left(W_{2}=0\right) f=1$. If we put in this case $\left\langle\left|Y_{21}(\omega)\right|^{2}\right\rangle_{\omega}=1$ we will obtain the instability condition of the isotropic phase with respect to the nematic phase formation [14, 40]. If we put $\left\langle\left|Y_{21}(\omega)\right|^{2}\right\rangle_{\omega}=1.1142$ we get the bifurcation of the nematic solution with the smallest value of the order parameter $S_{2}=0.3236$. Since this is the first order phase transition these two conditions are not equivalent. In the presence of an orienting field $\left(W_{2}>0\right)$ the fluid can exhibit only uniaxial paranematic and nematic phases. When $W_{2}<0$, the same fluid provides the phase transition into a biaxial nematic phase. At strong disorienting field $\left(W_{2} \rightarrow-\infty\right)$ the molecules align perpendicularly to the field and the phase transition into a limiting biaxial phase takes place [23]. In [24] it was shown that the fluid becomes orientationally unstable with respect to spontaneous biaxial nematic ordering under the condition

$$
1-\rho\left\langle\left|Y_{22}(\omega)\right|^{2}\right\rangle_{\omega} \rho \int C_{222}(r) \mathrm{d} \mathbf{r}=0 .
$$

This condition reduces to $(2.17)$ after $\left\langle\left|Y_{21}(\omega)\right|^{2}\right\rangle_{\omega}$ changes to $\left\langle\left|Y_{22}(\omega)\right|^{2}\right\rangle_{\omega}$. At the infinite field if we put $\left\langle\left|Y_{22}(\omega)\right|^{2}\right\rangle_{\omega}=\frac{15}{8}$ equation $(2.18)$ gives us the instability condition with respect to the limiting biaxial phase [23].

Figure 1 shows dependence of the order parameter $S_{2}$ on the product $\left(\beta a_{2} \eta\right)^{-1}$ calculated from (2.17) at different values of $z_{2} \sigma$. Here and below we consider that $A_{n}=a_{n} \sigma\left(z_{n} \sigma\right)^{2}$, where $n=0$ and 2. This allows us to consider the mean field result as the Kac potential limit $z_{2} \sigma \rightarrow 0$ and/or $z_{0} \sigma \rightarrow 0$ [41]. For $z_{2} \sigma=3$ and $\beta a_{2}=1, \eta$ has a non-physical value. As we will see later, in this region the system goes to crystallization. 


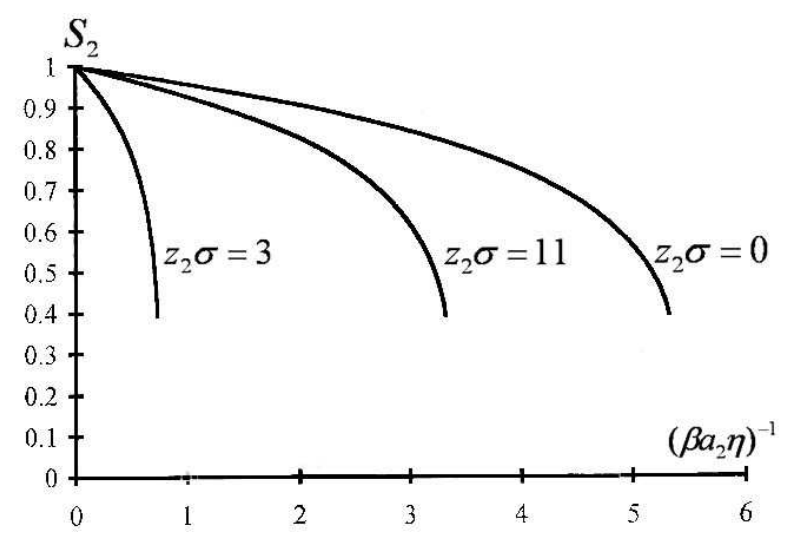

Figure 1. The dependence of the order parameter $S_{2}$ on density $\eta$ and temperature $\left(\beta a_{2}\right)^{-1}$ at different values of $z_{2} \sigma$ calculated in the MSA approximation for nematogenic Maier-Saupe model.

For $\mu=0$, using the factorization method of Wertheim-Baxter [18, 39], the integral equation (1.27) under the condition (1.32-1.33) can be reduced to the system of integral equations

$$
\begin{aligned}
& 2 \pi r C_{i j}(r)=-q_{i j}^{\prime}(r)+\sum_{k, l} \int \mathrm{d} t q_{i k}^{\prime}(r+t) \rho_{k l} q_{j l}(t), \\
& 2 \pi r h_{i j}(r)=-q_{i j}^{\prime}(r)+2 \pi \sum_{k, l} \int \mathrm{d} t(r-t) h_{i k}(|r-t|) \rho_{k l} q_{l j}(t) .
\end{aligned}
$$

It follows from the asymptotic behavior of the factor correlation functions that $q_{i j}(r)$ has the form $(i, j=0,2)$

$$
q_{i j}(r)=q_{i j}^{0}(r)+\sum_{n=0,2} D_{i j}^{(n)} \mathrm{e}^{-z_{n} r}
$$

where the short-range part

$$
q_{i j}^{0}(r)=\left\{\begin{array}{lc}
\frac{1}{2} q_{i j}^{\prime \prime}(r-\sigma)^{2}+q_{i j}^{\prime}(r-\sigma)+\sum_{n=0,2} C_{i j}^{(n)}\left(\mathrm{e}^{-z_{n} r}-\mathrm{e}^{-z_{n} \sigma}\right), r<\sigma, \\
0, & r>\sigma .
\end{array}\right.
$$

Below we shall use the following designations for dimensionless properties

$$
\begin{aligned}
c_{i j}^{(n)} & =\frac{\rho}{z_{n}} C_{i j}^{(n)}, \quad d_{i j}^{(n)}=\frac{\rho}{z_{n}} D_{i j}^{(n)}, \\
\tilde{g}_{i j}\left(z_{n}\right) & =\frac{\rho}{z_{n}} \int_{\sigma}^{\infty}\left[h_{i j}(t)+\delta_{i 0} \delta_{j 0}\right] \mathrm{e}^{-z_{n} t} \mathrm{~d} t, \\
\tilde{Q}_{i j}\left(z_{n}\right) & =\rho \int_{0}^{\infty} q_{i j}(t) \mathrm{e}^{-z_{n} t} \mathrm{~d} t .
\end{aligned}
$$

Finally, we obtain a system of algebraic equations for coefficients of factor correlation functions and Laplace transforms of a pair correlation function harmonics

$$
\begin{aligned}
-c_{i j}^{(n)} & =\sum_{l}\left[\delta_{i l}-2 \pi \sum_{k} \tilde{g}_{i k}\left(z_{n}\right) S_{k l}\right] d_{l j}^{(n)}, \\
\frac{12}{2 n+1} \beta A_{n} \frac{1}{\sigma} \eta \delta_{i n} \delta_{j n} & =\sum_{k} d_{i k}^{(n)}\left[\delta_{k j}-\sum_{l} S_{k l} \tilde{Q}_{j l}\left(z_{n}\right)\right],
\end{aligned}
$$




$$
\begin{gathered}
2 \pi \sum_{k} \tilde{g}_{i k}\left(z_{n}\right)\left[\delta_{k j}-\sum_{l} S_{k l} \tilde{Q}_{l j}\left(z_{n}\right)\right]=\frac{6}{\pi} \eta \mathrm{e}^{-z_{n} \sigma}\left[\frac{q_{i j}^{\prime \prime}}{\left(z_{n} \sigma\right)^{3}}+\frac{q_{i j}^{\prime}}{\left(z_{n} \sigma\right)^{2}}\right] \delta_{i 0} \\
-\sum_{m=0,2} \frac{z_{m}}{z_{m}+z_{n}} c_{i j}^{(m)} \exp \left(-\left(z_{m}+z_{n}\right) \sigma\right)
\end{gathered}
$$

where $\hat{S}=\frac{1}{\rho} \hat{\rho}, \hat{\rho}$ is given by (1.30).
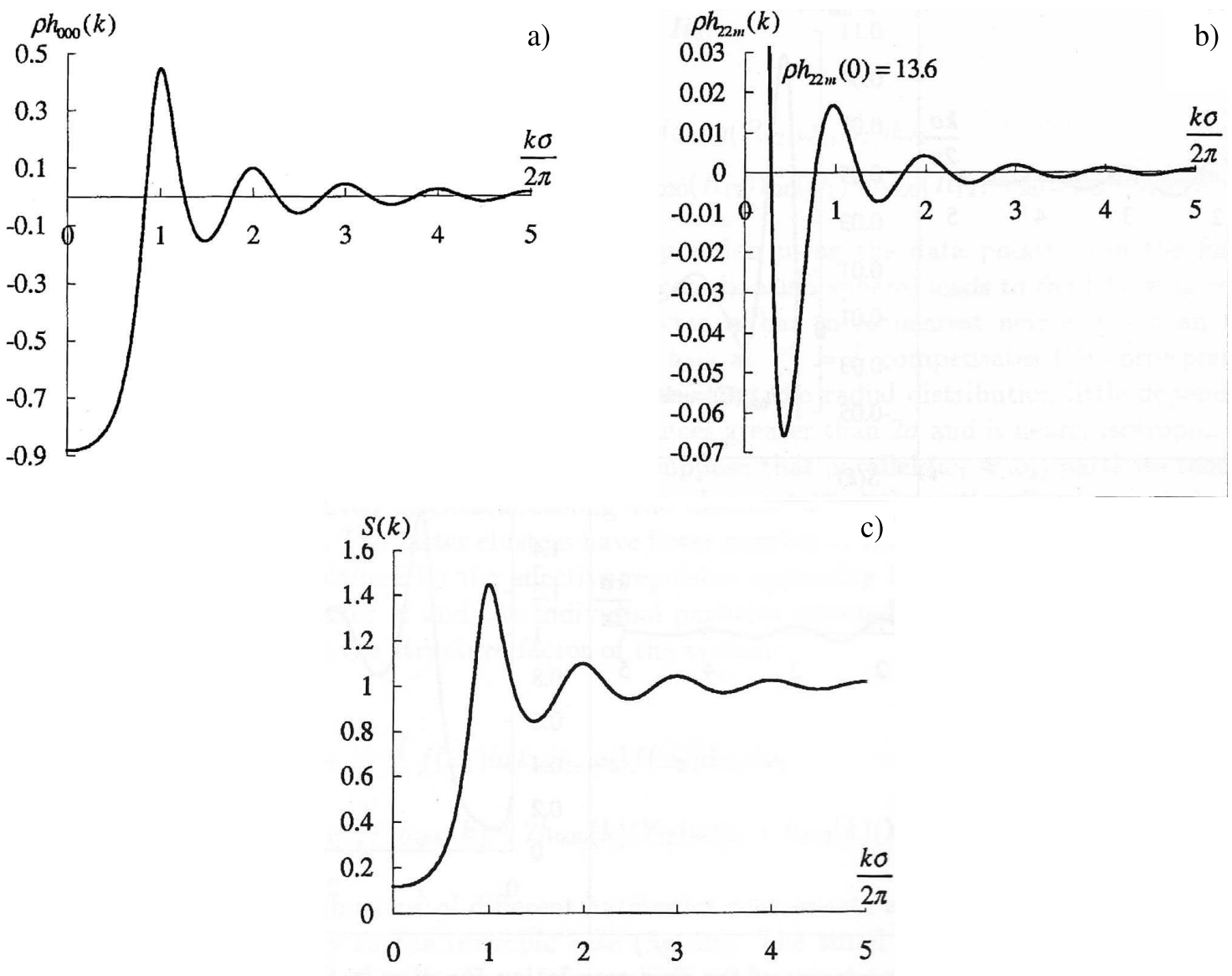

Figure 2. The harmonics of the pair correlation functions in the Fourier space and the structure factor for nematogenic Maier-Saupe model in isotropic phase $\left(z_{0} \sigma=z_{2} \sigma=1, \beta a_{0}=0.1, \beta a_{2}=\right.$ $1, \eta=0.28)$.

In (2.27) we should expect that multiple solutions occur, of which only one is acceptable [42]. To choose the physical solution one can utilize the condition

$$
\operatorname{det}[1-\hat{S} \hat{Q}(s)] \neq 0 \quad \text { for } \quad \operatorname{Re} s>0
$$

Using the obtained analytical solution of $\mathrm{OZ}$ equation for the considered model it is possible to calculate the structure factor and harmonics of the pair correlation functions. In figures 2 and 3 one can see the structure factor, and the Fourier-transforms of the pair correlation function harmonics $h_{m n \mu}(k)$ for the isotropic and nematic phases correspondingly in the absence of the external field. 


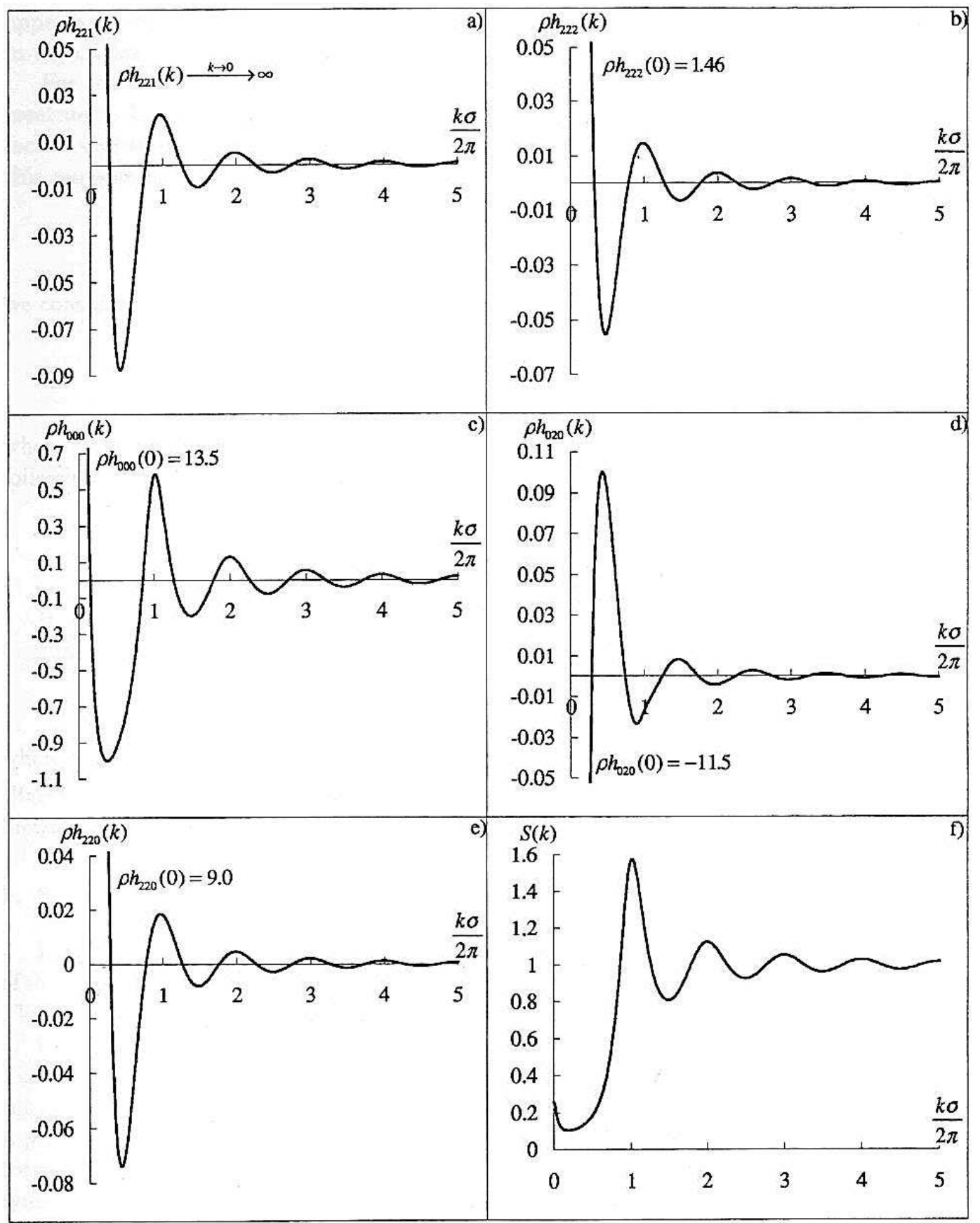

Figure 3. The harmonics of the pair correlation functions in the Fourier space and the structure factor for nematogenic Maier-Saupe model in the nematic phase $\left(z_{0} \sigma=z_{2} \sigma=1, \beta a_{0}=\right.$ $\left.0.1, \beta a_{2}=1, \eta=0.315\right)$.

The structure factor of the system

$$
\begin{aligned}
S(k) & =1+\rho \int f\left(\omega_{1}\right) h\left(k, \omega_{1}, \omega_{2}\right) f\left(\omega_{2}\right) \mathrm{d} \omega_{1} \mathrm{~d} \omega_{2} \\
& =1+\rho\left[h_{000}(k)+2 h_{020}(k)\left\langle Y_{20}(\omega)\right\rangle_{\omega}+h_{220}(k)\left\langle Y_{20}(\omega)\right\rangle_{\omega}^{2}\right] .
\end{aligned}
$$

We should note that in isotropic phase $h_{220}(k)=h_{221}(k)=h_{222}(k)$ and $h_{200}(k)=h_{020}(k)=0$. 
In the nematic phase the contributions of these harmonics are very important. One can see in figure 3 that in the nematic phase the off-diagonal elements $h_{200}(k)=h_{020}(k)$ at small $\mathrm{k}$ have comparable to $h_{220}(k)$ absolute value and opposite sign. Due to this the contributions of different harmonics into $S(k)$ compensate at small $\mathrm{k}$ and $S(k)$ in this region behaves similarly to isotropic case (figure 2 ). The small peak at small $\mathrm{k}$ in the nematic phase (figure 3 ) in $S(k)$ is attributed to the appearance of additional interparticle effective attraction due to parallel alignment of molecules. It is important to note that $h_{221}(k)$ is the only harmonic that tends to infinity at $k=0$ and this harmonic does not give any contribution to the structure factor which is finite at $k=0$.

Using equation (1.24) it is possible to show [14] that

$$
\rho\left\langle\left|Y_{21}(\omega)\right|^{2}\right\rangle_{\omega} h_{221}(k \rightarrow 0) \longrightarrow \frac{\left(z_{2} \sigma\right)^{2}}{(k \sigma)^{2}} \frac{4}{\left[\left(z_{2} \sigma\right)^{2} C \exp \left(-z_{2} \sigma\right)-2\right]^{2}}
$$

which implies the asymptotic behavior

$$
h_{221}(r \rightarrow \infty) \longrightarrow \frac{1}{6} \frac{\left(z_{2} \sigma\right)^{2}}{\left[\left(z_{2} \sigma\right)^{2} C \exp \left(-z_{2} \sigma\right)-2\right]^{2} \eta\left\langle\left|Y_{21}(\omega)\right|^{2}\right\rangle_{\omega}} \frac{\sigma}{r} .
$$

It can be shown that this harmonic is connected with the correlations of the director fluctuations. This result confirms the prediction from the fluctuation theory of de Gennes [18].

Now we consider the thermodynamic properties. The structure factor at $k=0$ gives us an isothermal compressibility

$$
\frac{1}{\beta}\left(\frac{\partial \rho}{\partial P}\right)_{T}=S(k=0) .
$$

The average energy of interparticle interaction at the absence of external field is calculated by

$$
\begin{aligned}
\frac{\beta \Delta E}{N}= & \beta 2 \pi \rho \int_{0}^{\infty} r^{2} \mathrm{~d} r \int \mathrm{d} \omega_{1} f\left(\omega_{1}\right) \int \mathrm{d} \omega_{2} f\left(\omega_{2}\right)\left[v_{0}(r)+v_{2}\left(r, \omega_{1}, \omega_{2}\right)\right] \\
& \times\left[g_{000}(r)+h_{200}(r) Y_{20}\left(\omega_{1}\right)+h_{020}(r) Y_{20}\left(\omega_{2}\right)+\sum_{\mu} h_{22 \mu}(r) Y_{2 \mu}\left(\omega_{1}\right) Y_{2 \mu}^{*}\left(\omega_{2}\right)\right] \\
= & 12 \eta \frac{\beta A_{0}}{\sigma}\left[g_{000}\left(z_{0} \sigma\right)+2 \sqrt{5} S_{2} h_{200}\left(z_{0} \sigma\right)+5 S_{2}^{2} h_{220}\left(z_{0} \sigma\right)\right]+12 \eta \frac{\beta A_{0}}{\sigma} \\
& \times\left[5 S_{2}^{2} g_{000}\left(z_{2} \sigma\right)+2 \sqrt{5} S_{2}\left\langle\left|Y_{20}(\omega)\right|^{2}\right\rangle_{\omega} h_{200}\left(z_{2} \sigma\right)+\sum_{\mu}\left(\left\langle\left|Y_{21}(\omega)\right|^{2}\right\rangle_{\omega}\right)^{2} h_{22 \mu}\left(z_{2} \sigma\right)\right] .
\end{aligned}
$$

Similarly, we can calculate the virial pressure

$$
\begin{aligned}
\frac{\beta \Delta P v}{\rho}= & -\beta \rho \frac{2}{3} \pi \int_{0}^{\infty} r^{3} \mathrm{~d} r \int \mathrm{d} \omega_{1} f\left(\omega_{1}\right) \int \mathrm{d} \omega_{2} f\left(\omega_{2}\right)\left[g_{000}(r)+h_{200}(r) Y_{20}\left(\omega_{1}\right)\right. \\
& \left.+h_{020}(r) Y_{20}\left(\omega_{2}\right)+\sum_{\mu} h_{22 \mu}(r) Y_{2 \mu}\left(\omega_{1}\right) Y_{2 \mu}^{*}\left(\omega_{2}\right)\right] \frac{\partial}{\partial r}\left[v_{h}(r)+v_{0}(r)+v_{2}\left(r, \omega_{1} \omega_{2}\right)\right] \\
= & 4 \eta\left[g_{000}(\sigma+)+2 \sqrt{5} S_{2} h_{200}(\sigma+)+5 S_{2}^{2} h_{220}(\sigma+)\right]+\frac{1}{3} \frac{\beta \Delta E}{N} \\
& +4 \eta \frac{\beta A_{0}}{\sigma} z_{0} \frac{\partial}{\partial z_{0}}\left[g_{000}\left(z_{0} \sigma\right)+2 \sqrt{5} S_{2} h_{200}\left(z_{0} \sigma\right)+5 S_{2}^{2} h_{220}\left(z_{0} \sigma\right)\right] \\
& +4 \eta \frac{\beta A_{2}}{\sigma} z_{2} \frac{\partial}{\partial z_{2}}\left[5 S_{2}^{2} g_{000}\left(z_{2} \sigma\right)+2 \sqrt{5} S_{2}\left\langle\left|Y_{20}(\omega)\right|^{2}\right\rangle_{\omega} h_{200}\left(z_{2} \sigma\right)\right. \\
& \left.+\sum_{\mu}\left(\left\langle\left|Y_{21}(\omega)\right|^{2}\right\rangle_{\omega}\right)^{2} h_{22 \mu}\left(z_{2} \sigma\right)\right],
\end{aligned}
$$


where $g_{000}(r)=1+h_{000}(r), g_{000}\left(z_{n} \sigma\right)$ and $h_{m n \mu}\left(z_{n} \sigma\right)$ are Laplace-transforms of corresponding functions at $z_{n} \sigma$.
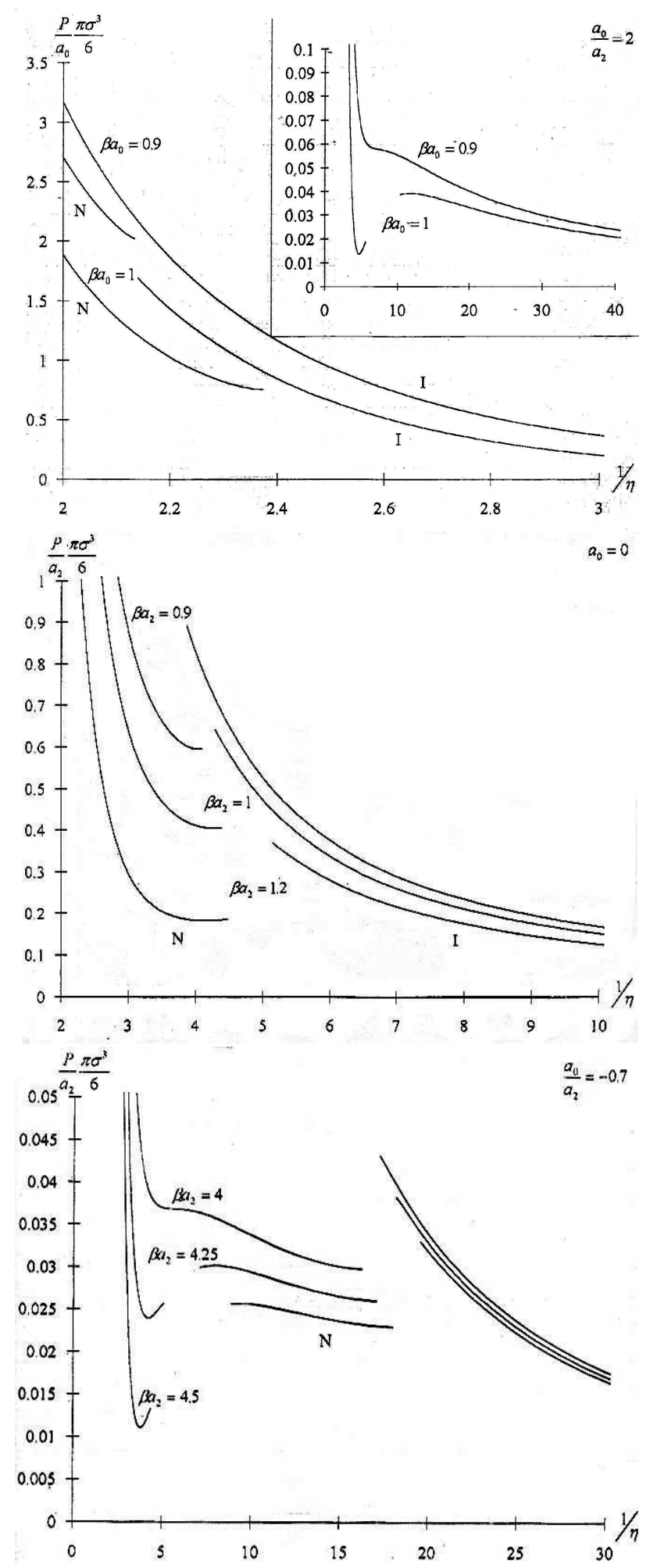

Figure 4. Some isotherms of equation of state for nematogenic Maier-Saupe model. 
Some isotherms calculated using the equation of state (2.35) are presented in figure 4 for three different regimes [43]: 1 . The isotropic attraction is stronger than the anisotropic one $\left(\frac{a_{0}}{a_{2}}=2\right)$; 2. Isotropic attraction is absent $\left(a_{0}=0\right) ; 3$. Strong anisotropic attraction and isotropic repulsion $\left(\frac{a_{0}}{a_{2}}=-0.7\right)$. For simplification we consider that $z_{0} \sigma=z_{2} \sigma=0.5$. Nematic and isotropic branches are denoted by $\mathrm{N}$ and I correspondingly. At the first case when isotropic attraction is stronger than the anisotropic one at smaller densities and lower temperature $\left(\beta a_{0}=1\right)$ there is condensation between two isotropic phases which disappears at high temperature $\left(\beta a_{0}=0.9\right)$. In the second case when isotropic attraction is absent at high temperature $\left(\beta a_{2}=0.9\right)$ we observe the weak isotropicnematic phase transition. At the lower temperature $\left(\beta a_{2}=1\right)$ we observe condensation in the nematic region. In the third case $\left(\frac{a_{0}}{a_{2}}=-0.7\right)$ at the lower temperature $\left(\beta a_{2}=4.25\right)$ we observe the liquid-gas phase transition between two nematic phases. The entire liquid-gas coexistence region including the critical point is within the nematic region.

For the description of phase diagram we need to have the expression for the chemical potential of fluid which can be obtained by generalization of the Hoye-Stell scheme [44]. Unfortunately, this problem has nor been solved yet. We will consider it in a separate paper. Here we will instead use the density functional scheme developed by us in [20] for the chemical potential and the expression (2.35) for the pressure. For simplification we consider the case $a_{0}=0$.
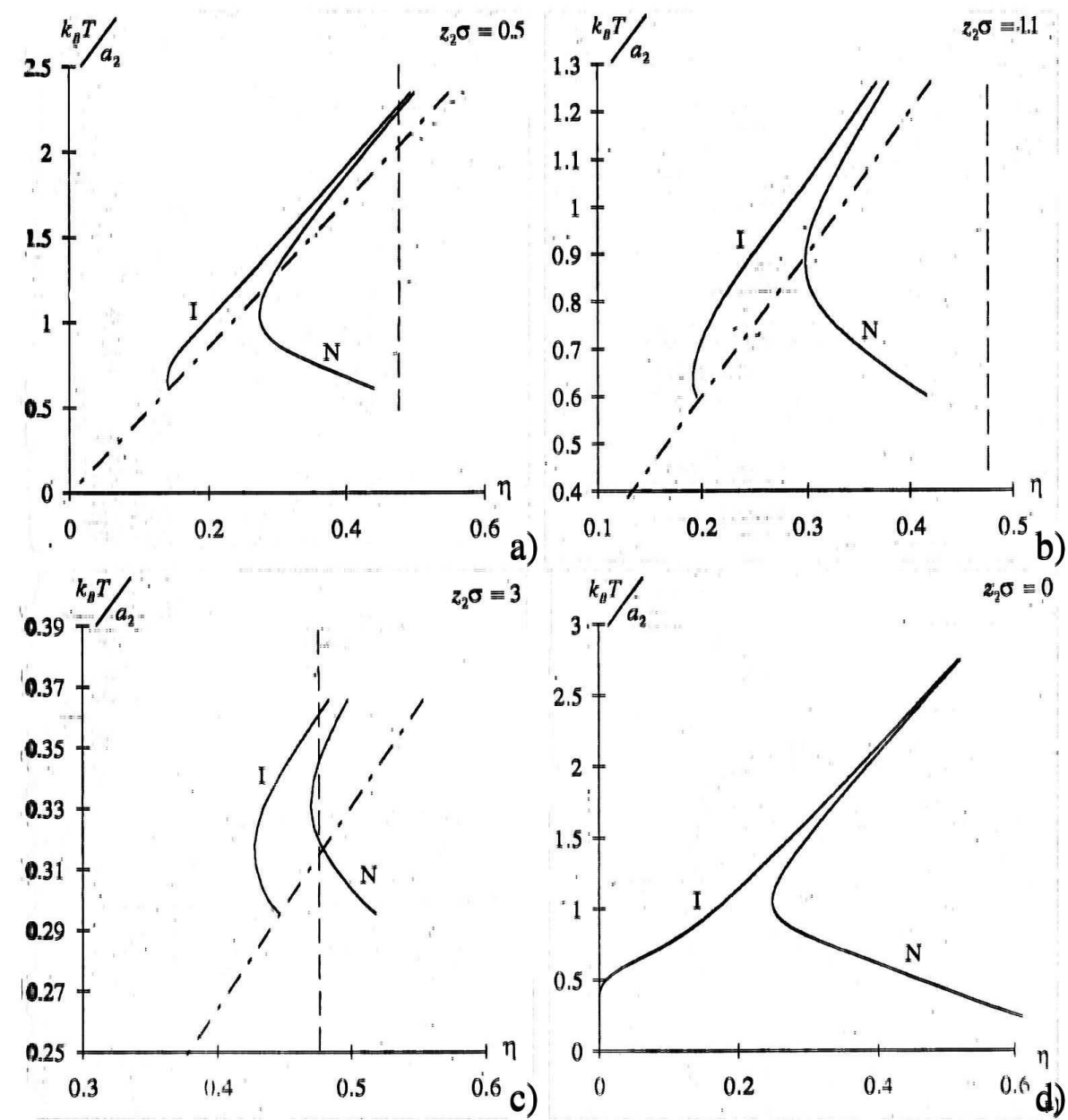

Figure 5. Phase diagram of nematogenic Maier-Saupe model for different values of $z_{2} \sigma$ in the plane density-temperature. 


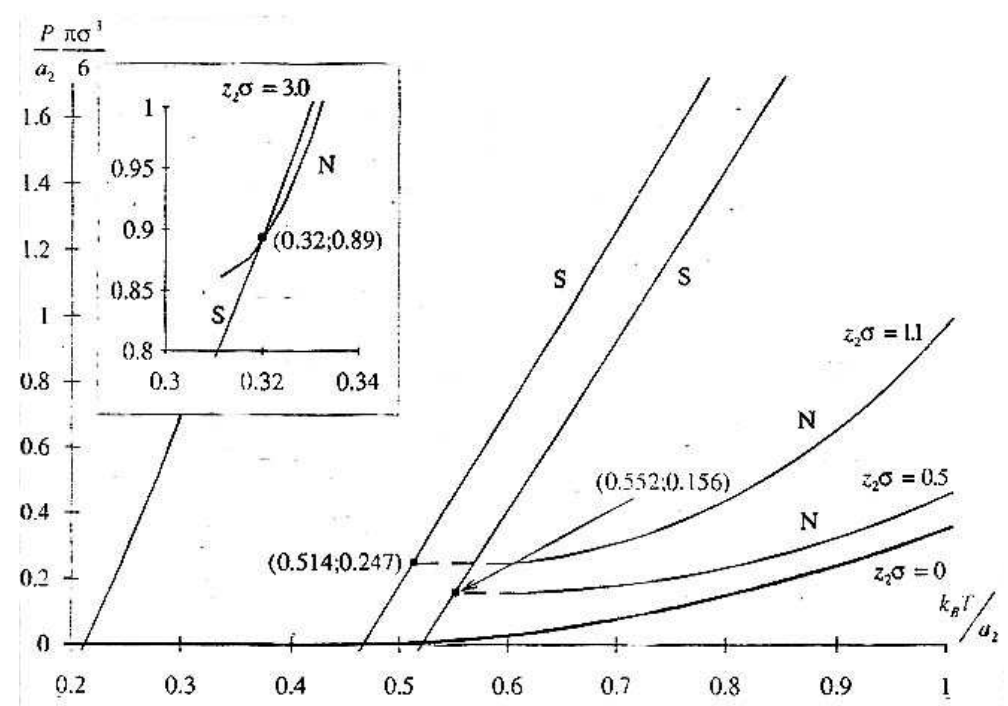

Figure 6. Phase diagram of the nematogenic Maier-Saupe model for different values of $z_{2} \sigma$ in the plane temperature-pressure.

In figures 5 and 6 we present a phase diagram for the considered model at the planes $\eta-$ $\frac{k T}{a_{2}}$ (density-temperature) and $\frac{k T}{a_{2}}-\frac{P \eta}{\rho a_{2}}$ (temperature-pressure) at different $z_{2} \sigma$. Dash-dotted line corresponds to the stability condition for the isotropic phase. As we can see, this condition overestimates the region of anisotropic phase. This overestimation increases with the increase of $z_{2} \sigma$. But we should take into account that with the increase of $z_{2} \sigma$ the accuracy of MSA decreases. For $z_{2} \sigma=0.5$ at high temperature we observed a weak nematic transition of the first order. With decreasing temperature, the jump of density at the phase transition increases and at low temperature the orientational order is accompanied by condensation. The peculiarity of this condensation is that it occurs without a critical point. It means that there is no phase transition "nematic - condensed nematic". In figure 7 the temperature dependence of the order parameter at the phase transition region is presented. In figure 5 the dotted line represents the crystallization

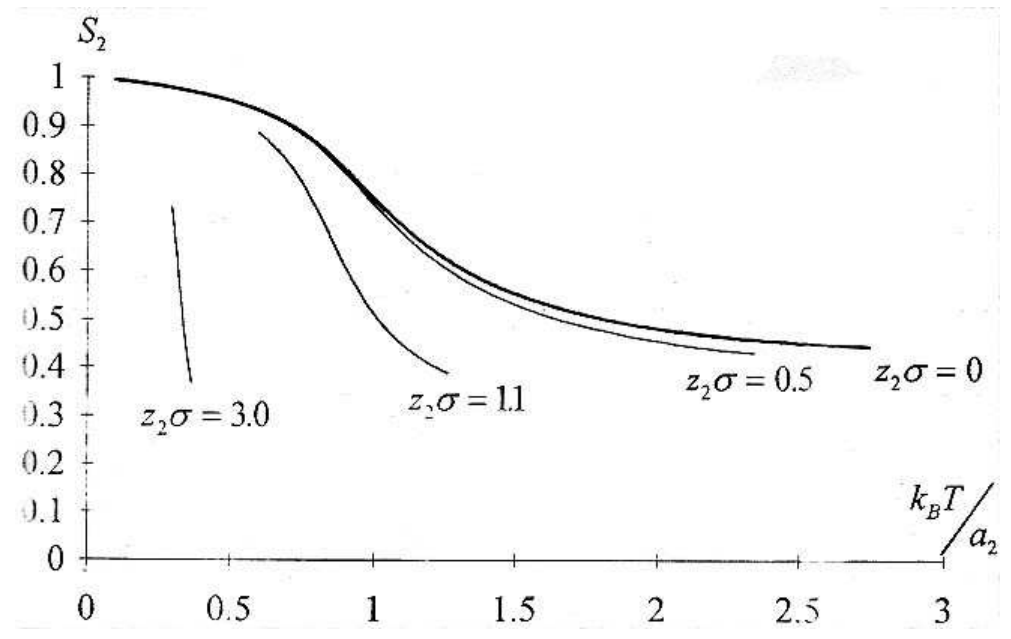

Figure 7. Temperature dependence of the order parameter $S_{2}$ at the phase transition region for the nematic phase. 
transition line. It was obtained using the Hansen-Verlet criterion [45]. According to this criterion the fluid becomes unstable when the height of the main peak in the structure factor $S(k)$ becomes equal to $2.9 \pm 0.1$. Figure 6 gives evidence of the existence of temperature at which three phases coexist (isotropic, nematic, and solid). As we can see with the increase of $z_{2} \sigma$, the triple point shifts to the region of higher pressure and lower temperature. Since with the increase of temperature the density of crystallization increases we can see that at high enough temperature the crystallization can forestall the nematic transition. We can note that for not so large value of $z_{2} \sigma$ the phase diagram presented in figure 5 agrees quite well with the results of [16] obtained in the framework reference $\mathrm{HNC}$ and from computer simulation.
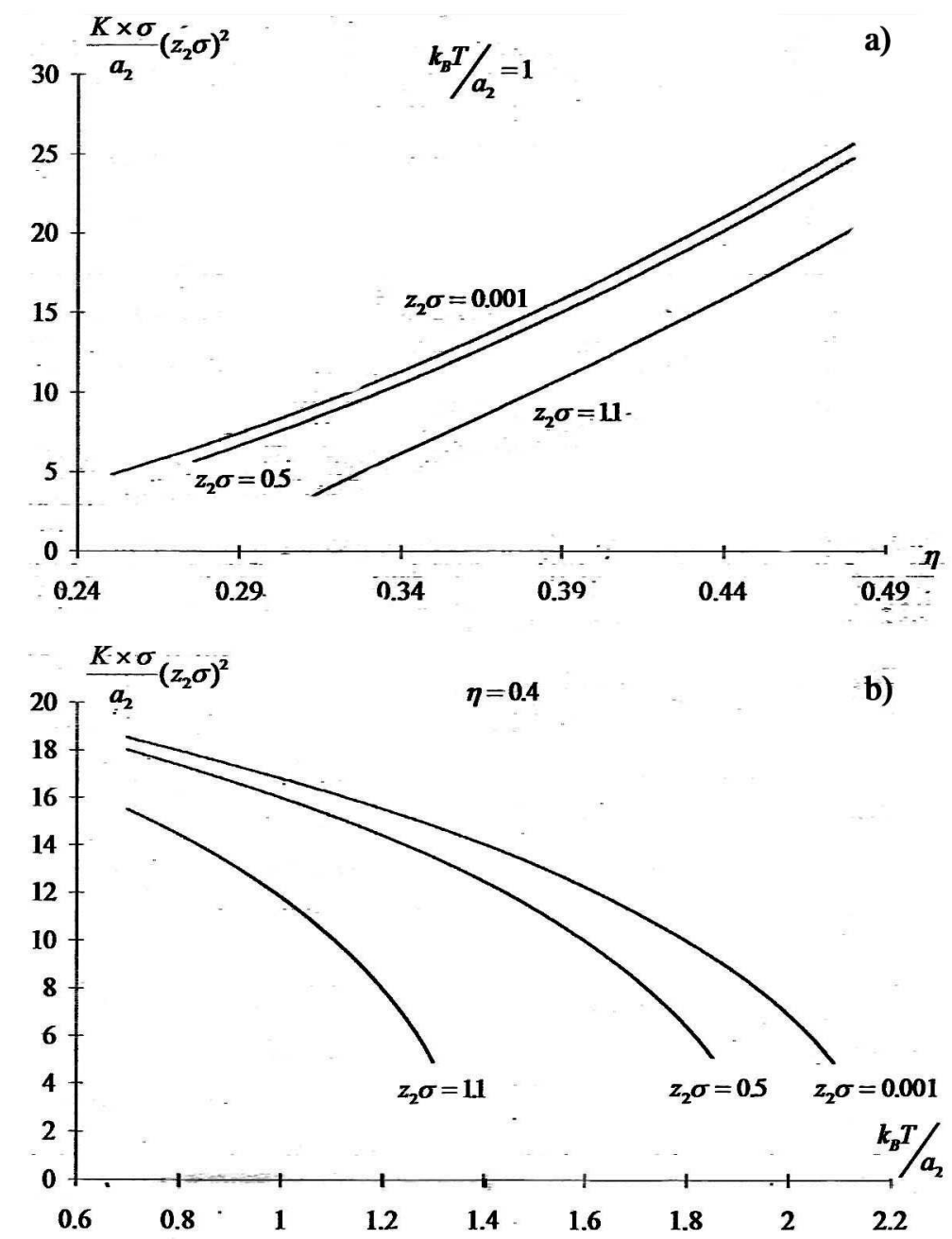

Figure 8. The dependence of reduced elastic constants on density and temperature.

Let us consider the elastic properties of the considered model. Formal expressions for elastic constant in biaxial nematics in terms of direct correlation function have been given by Poniewiersky and Stecki [46]. It includes three elastic constants $K_{1}$ (splay), $K_{2}$ (twist) and $K_{3}$ (bend) [47]. Since for the considered model correlation functions depend only on the angle $\omega_{1} 2$, the description of elastic properties reduces to one-constant approximation

$$
\beta K_{1}=\beta K_{2}=\beta K_{3}=\beta K=\frac{1}{6} \rho^{2} \int \mathrm{d} \mathbf{r} \mathrm{d} \omega_{1} \mathrm{~d} \omega_{2} r^{2} \dot{f}\left(\omega_{1}\right) \dot{f}\left(\omega_{2}\right) n_{x}\left(\omega_{1}\right) n_{x}\left(\omega_{2}\right) C_{2}\left(r, \omega_{1} \omega_{2}\right)
$$


where $\dot{f}\left(\omega_{1}\right)=\frac{\partial f(\omega)}{\partial \cos \vartheta}$.

In the MSA approximation

$$
\beta K=10 \pi \rho^{2} S_{2}^{2} \int r^{4} \mathrm{~d} \mathbf{r} C_{221}(r) .
$$

Another way of calculating the elastic constants is connected with the application of the theory of hydrodynamic fluctuations [46]. In this way in one-constant approximation

$$
\frac{1}{\beta K}=\frac{1}{3} \lim _{k \rightarrow 0} k^{2} h_{221}(k) \frac{\left\langle\left|Y_{21}(\omega)\right|^{2}\right\rangle_{\omega}}{\left\langle\left|Y_{20}(\omega)\right|\right\rangle_{\omega}^{2}} .
$$

The results of our calculations are presented in figure 8. It is important to note that in our calculations both expressions (2.37) and (2.38) give the same results.

The effect of a disorienting field on the phase diagram and on the elastic properties of the ordered fluids was studied by us in $[23,24]$. It was shown that a disorienting field significantly increases the region of an ordered fluid. In the case of a strong disorienting field when the temperature decreases the orientational phase transition of the second order becomes a transition of the first order at a tricritical point. A disorienting field increases the ordering and the elastic properties of the model under consideration.

\section{Application of the integral equation theory to colloid-nematic dispersi- ons}

In this section we review the results of recent publications of T. Sokolovska, R. Sokolovskii and G. Patey 28 35] about the generalization of the integral equation theory for colloid-nematic systems. The starting point of this generalization is the $\mathrm{OZ}$ equations for a two-component mixture of colloidal and nematic particles

$$
\begin{aligned}
& h_{\mathrm{CC}}(12)=C_{\mathrm{CC}}(12)+\int \mathrm{d} 3 \rho_{\mathrm{C}}(3) C_{\mathrm{CC}}(13) h_{\mathrm{CC}}(32)+\int \mathrm{d} 3 \rho_{\mathrm{N}}(3) C_{\mathrm{CN}}(13) h_{\mathrm{CN}}(32), \\
& h_{\mathrm{CN}}(12)=C_{\mathrm{CN}}(12)+\int \mathrm{d} 3 \rho_{\mathrm{C}}(3) C_{\mathrm{CC}}(13) h_{\mathrm{CN}}(32)+\int \mathrm{d} 3 \rho_{\mathrm{N}}(3) C_{\mathrm{CN}}(13) h_{\mathrm{NN}}(32), \\
& h_{\mathrm{NN}}(12)=C_{\mathrm{NN}}(12)+\int \mathrm{d} 3 \rho_{\mathrm{C}}(3) C_{\mathrm{NC}}(13) h_{\mathrm{CN}}(32)+\int \mathrm{d} 3 \rho_{\mathrm{N}}(3) C_{\mathrm{NN}}(13) h_{\mathrm{NN}}(32)
\end{aligned}
$$

in combination with TZLMBW equations for density distributions of colloidal and nematic particles, respectively

$$
\begin{aligned}
& \beta \nabla v_{\mathrm{C}}(1)+\nabla \ln \rho_{\mathrm{C}}(1)=\int \mathrm{d} 2 C_{\mathrm{CC}}(12) \nabla \rho_{\mathrm{C}}(2)+\int \mathrm{d} 2 C_{\mathrm{CN}}(12) \nabla \rho_{\mathrm{N}}(2), \\
& \beta \nabla v_{\mathrm{N}}(1)+\nabla \ln \rho_{\mathrm{N}}(1)=\int \mathrm{d} 2 C_{\mathrm{NC}}(12) \nabla \rho_{\mathrm{C}}(2)+\int \mathrm{d} 2 C_{\mathrm{NN}}(12) \nabla \rho_{\mathrm{N}}(2),
\end{aligned}
$$

where the label 1 denotes the coordinates $\left(\mathbf{r}_{1}, \omega_{1}\right)$ for nematogen and for spherical colloids $1=\left(\mathbf{r}_{1}\right)$.

Here we consider a dilute nematic colloids case for which OZ equations (3.1)-(3.3) reduce to

$$
\begin{aligned}
& h_{\mathrm{NN}}(12)=C_{\mathrm{NN}}(12)+\int \mathrm{d} 3 \rho_{\mathrm{N}}(3) C_{\mathrm{NN}}(13) h_{\mathrm{NN}}(32), \\
& h_{\mathrm{CN}}(12)=C_{\mathrm{CN}}(12)+\int \mathrm{d} 3 \rho_{\mathrm{N}}(3) C_{\mathrm{CN}}(13) h_{\mathrm{NN}}(32), \\
& h_{\mathrm{CC}}(12)=C_{\mathrm{CC}}(12)+\int \mathrm{d} 3 \rho_{\mathrm{N}}(3) C_{\mathrm{CN}}(13) h_{\mathrm{NC}}(32)
\end{aligned}
$$

in combination with the usual TZLMBW equation (1.18) for a nematic subsystem

$$
\beta \nabla_{\omega_{1}} v_{\mathrm{N}}(1)+\nabla_{\omega_{1}} \ln \rho_{\mathrm{N}}(1) \int \mathrm{d} 2 C_{\mathrm{NN}}(12) \nabla_{\omega_{2}} \rho_{n}(2) .
$$


Equation (3.6) coincides with equation (1.6) for bulk nematic fluids. Equation (3.7) describes nematic fluids near colloidal particles. The function $\rho_{\mathrm{N}}(1)\left[1+h_{\mathrm{NC}}(12)\right]$ gives distribution of a nematic fluid about a colloidal particle. This function takes into account all the changes at a given point $\mathbf{r}_{1}$ induced by a colloidal particle at the point $\mathbf{r}_{2}$. These include the changes in the local density and in the orientational distribution of the nematic fluid. Equation (3.8) describes the colloid-colloid correlations. It gives the colloid-colloid mean interaction force which at the HNC level is conveniently given by

$$
\beta w_{\mathrm{CC}}(12)=\beta v_{\mathrm{CC}}(12)+C_{\mathrm{CC}}(12)-h_{\mathrm{CC}}(12)
$$

where $v_{\mathrm{CC}}(12)$ is the direct pair interaction potential between colloidal particles.

For nematic we consider the same model as in the previous sections. This is the model of hard spheres with an anisotropic interaction in the form (1.2). For simplification we put here $A_{0}=0$. The nematogen interaction with the external field is given by (1.1). The model colloidal particles (C) are taken to be hard spheres of diameter R. Van der Waals or other direct colloid-colloid interactions could be included through the $v_{\mathrm{CC}}(12)$ term in equation (3.10). We consider the size of a colloid to be much larger than the size of a nematic particle. The properties of a nematogenic fluid near the surface can then be described in the Henderson-Abraham-Barker (HAB) approach 27]. This approach reduces to equation (3.7) in the limit $R \rightarrow \infty$. In this case we can switch from the colloid-nematic distance to the colloid-surface distance $s_{12}$. The interaction of nematogens with the surface of a colloidal particle (anchoring) is modeled as was done for the flat wall case [28, 29]

$$
v_{\mathrm{NC}}(12)=\left\{\begin{array}{cl}
-A_{\mathrm{C}} \exp \left[-z_{\mathrm{C}}\left(s_{12}-\frac{1}{2} \sigma\right)\right] P_{2}\left(\omega, \hat{s}_{12}\right) & \text { for } \quad s_{12}>\frac{1}{2} \sigma \\
\infty, & \text { for } \quad s_{12}<\frac{1}{2} \sigma
\end{array}\right.
$$

where $\mathbf{s}_{12}$ is the vector connecting the nearest point of the surface of colloid 1 with the center of nematogen 2 , and $\hat{s}_{12}=\frac{\mathbf{s}_{12}}{s_{12}}$. Note that the positive and the negative values of $A_{\mathrm{C}}$ favor respectively the perpendicular and the parallel orientations of nematogen molecules with respect to the surface. The strength of the nematogen-colloid interaction is determined by $A_{\mathrm{C}}$ and $z_{\mathrm{C}}$.

We start with solving the $\mathrm{OZ}$ equation (3.7) for the wall-nematic correlation function with the MSA closure

$$
\begin{array}{ll}
h_{\mathrm{WN}}\left(\hat{s} \mid \hat{\omega}_{1}, s_{1}\right)=-1, & s_{1}<\frac{1}{2} \sigma, \\
C_{\mathrm{WN}}\left(\hat{s} \mid \hat{\omega}_{1}, s_{1}\right)=-\beta v_{\mathrm{WN}}\left(\hat{s} \mid \hat{\omega}_{1}, s_{1}\right), & s_{1}>\frac{1}{2} \sigma .
\end{array}
$$

After application to correlation functions $h_{\mathrm{WN}}$ and $C_{\mathrm{WN}}$ orientational harmonics expansion

$$
f_{\mathrm{WN}}\left(\hat{s} \mid \hat{\omega}_{1}, s_{1}\right)=f_{000}^{\mathrm{WN}}\left(s_{1}\right)+f_{200}^{\mathrm{WN}}\left(s_{1}\right) Y_{20}(\hat{s})+f_{020}^{\mathrm{WN}}\left(s_{1}\right) Y_{20}\left(\omega_{1}\right)+\sum_{\mu} f_{22 \mu}^{\mathrm{WN}}\left(s_{1}\right) Y_{2 \mu}\left(\hat{s}_{1}\right) Y_{2 \mu}\left(\omega_{1}\right) .
$$

OZ equation (3.7) reduces to the system of equations for harmonics coefficients $f_{m n \mu}^{\mathrm{WN}}\left(s_{1}\right)$. For $\mu \neq 0$

$$
h_{22 \mu}^{\mathrm{WN}}\left(s_{1}\right)=C_{22 \mu}^{\mathrm{WN}}\left(s_{1}\right)+\rho\left\langle\left|Y_{2 \mu}(\omega)\right|^{2}\right\rangle_{\omega} \int h_{22 \mu}^{\mathrm{WN}}\left(s_{2}\right) C_{22 \mu}^{\mathrm{NN}}\left(r_{12}\right) \mathrm{d} \mathbf{r}_{2}
$$

and for $\mu=0$

$$
h_{i j 0}^{\mathrm{WN}}\left(s_{1}\right)=C_{i j 0}^{\mathrm{WN}}\left(s_{1}\right)+\int \mathrm{d} \mathbf{r}_{2} \rho \sum_{i^{\prime} j^{\prime}} h_{i i^{\prime}, 0}^{\mathrm{WN}}\left(s_{2}\right)\left\langle\left|Y_{i^{\prime} 0}(\omega) Y_{j 0}(\omega)\right|\right\rangle_{\omega} C_{j^{\prime} j 0}^{\mathrm{NN}}\left(r_{12}\right)
$$

where the indices can be 0 or 2 .

To solve equations (3.14) and (3.15) we can directly follow the Baxter-Wertheim factorization technique developed for calculating the wall-particle distribution functions [48]. As a result, for 
$s>\frac{1}{2} \sigma$ and for $\mu=0$, equations (3.14) and (3.15) can be written correspondingly in the form

$$
\hat{g}^{\mathrm{WN}}(s)-\int_{0}^{\infty} \hat{g}^{\mathrm{WN}}(s-r) \hat{\rho} \hat{q}(r) d r=\hat{v}_{\mathrm{WN}}\left[1-\hat{q}^{T}\left(z_{\mathrm{C}}\right)\right]^{-1} \mathrm{e}^{-z_{\mathrm{C}} s}+\left(\begin{array}{ll}
1 & 0 \\
0 & 0
\end{array}\right)[1-\hat{q}(s=0)]
$$

and for $\mu \neq 0$

$$
h_{22 \mu}^{\mathrm{WN}}(s)=\rho\left\langle\left|Y_{2 \mu}(\omega)\right|^{2}\right\rangle_{\omega} \int_{0}^{\infty} h_{22 \mu}^{\mathrm{WN}}(s-r) q_{22 \mu}(r) \mathrm{d} r+\frac{1}{5} \beta A_{\mathrm{C}} \frac{\exp \left[z_{\mathrm{C}}\left(\frac{1}{2} \sigma-s\right)\right]}{1-\rho\left\langle\left|Y_{2 \mu}(\omega)\right|^{2}\right\rangle_{\omega} q_{22 \mu}\left(z_{\mathrm{C}}\right)}
$$

where the matrix $\hat{g}_{\mathrm{WN}}(s)$ has the elements $g_{i j}^{\mathrm{WN}}(s)=\delta_{i 0} \delta_{j 0}+h_{i j}^{\mathrm{CN}}(s), \hat{v}_{\mathrm{WN}}$ has the elements $v_{i j}^{\mathrm{WN}}=\frac{1}{5} \beta A_{\mathrm{C}} \delta_{i 2} \delta_{j 2} \exp \left[\frac{1}{2} \sigma z_{\mathrm{C}}\right]$. The matrices $\hat{\rho}$ and Baxter functions $\hat{q}(r)$ and $q_{22 \mu}(r)$ and its Laplace transforms $q\left(z_{\mathrm{C}}\right)$ are discussed in previous sections. The right-hand sides of equations (3.16) and (3.17) determine the contact values of $\hat{g}^{\mathrm{WN}}\left(\frac{1}{2} \sigma\right)$ and $h_{22 \mu}^{\mathrm{WN}}\left(\frac{1}{2} \sigma\right)$ for $\mu \neq 0$. One can solve equations (3.16) and (3.17) at any distance $s$ using the Perram method [47], for example.

The wall-nematic distribution $g^{\mathrm{WN}}(12)$ in the form $(3.13)$ can be used to examine the structure near the wall. Using this distribution function it is reasonable to introduce the density profile which is defined as

$$
\rho(\hat{s} \mid s)=\int g_{\mathrm{CN}}\left(\hat{s}_{1} \mid \omega_{1} s_{1}\right) \rho_{\mathrm{N}}\left(\omega_{1}\right) \mathrm{d} \omega_{1} .
$$

In contrast to the usual fluids, the nematic density profile depends not only on the distance from the wall $s_{1}$, but also on the wall orientation $\hat{s}$.

Noting the tensorial nature of orientational ordering near the wall, it is useful to define a generalized order parameter

$$
S(\hat{d})=\int P_{2}(\hat{\omega} \cdot \hat{d}) \rho_{\mathrm{N}}(\hat{\omega}) \mathrm{d} \hat{\omega}
$$

where $\hat{d}$ is an arbitrary unit vector. For the considered nematic model the unit vector $\hat{d}_{m}$ that maximizes $S(\hat{d})$ is chosen as a director. In the presence of an aligning field the bulk director is always parallel to this field and $S\left(\hat{d}_{m}\right) / \rho=S_{2}$. The density-orientational profile of the generalized order parameter can be defined as

$$
S\left(\hat{s}_{1}, s_{1}, \hat{d}\right)=\int P_{2}\left(\hat{\omega}_{1} \hat{d}\right) \rho_{\mathrm{N}}\left(\omega_{1}\right) g^{\mathrm{WN}}\left(\hat{s}_{1}, s_{1}, \omega_{1}\right) \mathrm{d} \omega_{1} .
$$

For a large distance from the wall $s_{1}$ the distribution function $g^{\mathrm{CN}}\left(\hat{s}_{1} s_{1}, \omega_{1}\right)$ tends to 1 and $S(\hat{d})$ tends to the bulk value $S(\hat{d})=\rho s_{2} P_{2}(\hat{d} \hat{n})$. By analogy with the bulk definition, the unit vector $\hat{d}_{m}$ that maximizes $S(s, \hat{s}, d)$ at a given distance $s_{1}$ can be taken to define a local director. The vector field $d_{m}\left(s_{1}, \hat{s}\right)$ gives the director field configuration (the defect) around the wall. The maximum value $S_{m}\left(s_{1}\right)=S\left(s_{1}, d_{m}\right)$ gives the degree of local ordering at $s_{1}$.

From the investigation of [28, 29], for an orienting wall special long-range correlations were identified that are responsible for the reorientation of the bulk nematic at the zero external field. These correlations become stronger as the wall-nematic interaction is increased in range. They become longer ranged as the orienting field is weakened. The local director orientation can vary discontinuously with the distance from the wall when the orienting effect of the field and wall-nematic interaction are antagonistic. At high densities, when wall-nematic interaction favors orientations perpendicular to the surface, smectic-like structures were observed.

An important property that can be calculated from (3.18) is the adsorption coefficient which describes the surface density excess. In contrast to usual fluids, for anisotropic fluids the adsorption coefficient depends on the surface orientation $\hat{s}$ with respect to the external field and has the form

$$
\Gamma(\hat{s})=\rho \int_{\frac{1}{2} \sigma}^{\infty} \mathrm{d} s_{1} \int \mathrm{d} \omega_{1} f_{\mathrm{N}}\left(\omega_{1}\right)\left[g^{\mathrm{CN}}\left(s, \omega_{1}, s_{1}\right)-1\right] .
$$


Above we introduced a generalized order parameter (3.20) related to a certain direction $\hat{d}$. The adsorption excess per unit area associated with this order parameter is given by

$$
\Gamma(\hat{s}, \hat{d})=\rho \int_{\frac{\sigma}{2}}^{\infty} \mathrm{d} s_{1} \int \mathrm{d} \omega_{1} P_{2}(\hat{d} \cdot \hat{\omega})\left[g^{\mathrm{WN}}\left(\hat{s}_{1} \hat{\omega}, s_{1}\right)-1\right] f\left(\omega_{1}\right) .
$$

The maximum of this function gives information about the direction in which particles are mostly reoriented, and the minimum indicates the direction which they mostly abandon.

There is a significant flaw in the HAB description of the MSA approach due to ignoring the non-direct interaction between colloid and nematic in the case of the inert hard wall $\left(A_{\mathrm{C}}=0\right)$. This problem has recently been solved in the framework of the inhomogeneous equation theory [50] (the so-called OZ2 approach [3]).

Now we can return to colloid-nematic systems described by equations (3.7)-(3.8). To this end, we can use the results that had been obtained for a nematic near hard wall. We need to introduce the center-center colloid-molecule vector $\mathbf{r}_{12}$ which is parallel to $\hat{s}_{1}$ and whose length is $s_{1}+\frac{1}{2} R$, where $R$ is the diameter of the colloidal particle. For example, the colloid-nematogen interaction $v^{\mathrm{CN}}(12)$ can be expressed in terms of the vector connecting the nearest point on the surface of colloidal particle 1 with the center of nematogen 2. This transformation is the simplest for a spherical colloid

$$
\begin{aligned}
v^{\mathrm{CN}}(12) & =v^{\mathrm{CN}}\left(\mathbf{r}_{12}, \hat{\omega}_{2}\right)=v^{\mathrm{WN}}\left(\mathbf{s}=\mathbf{r}_{12}-\frac{\hat{\mathbf{r}}_{12}}{2} R, \hat{\omega}=\hat{\omega}_{2}\right) \\
& =-A_{\mathrm{C}} \exp \left[-z_{\mathrm{C}}\left(r_{12}-\frac{1}{2}(\sigma+R)\right)\right] P_{2}\left(\hat{r}_{12} \cdot \hat{\omega}_{2}\right) .
\end{aligned}
$$

For a sufficiently large colloidal particle, curvature effects are unimportant (for micron and submicron colloids), which suggests an ansatz that the direct correlation function $C_{\mathrm{CN}}(12)$ can be taken from the wall-nematic solution

$$
C^{\mathrm{CN}}\left(\mathbf{r}_{12}, \hat{\omega}_{2}\right)=C^{\mathrm{WN}}\left(\mathbf{s}=\mathbf{r}_{12}-\frac{\hat{\mathbf{r}}_{12}}{2} R, \hat{\omega}=\hat{\omega}_{2}\right) .
$$

This was found to be a good approximation 31, 32] because the wall-nematic direct correlation function is truly short-ranged outside the surface, while inside the core it rapidly tends to a function of $\hat{\omega}_{2}$ that depends only on bulk properties. Thus, the direct correlation function is not very sensitive to the surface curvature.

Now the nematic distribution around a colloidal particle and colloid-colloid mean force potential can be found from equations (3.7) and (3.9) by means of the Fourier transformation. As we already discussed in the previous section, the correlation functions $C^{\mathrm{NN}}(12)$ and $h^{\mathrm{NN}}(12)$ in the MSA approximation can be presented in the form (1.21) and the corresponding OZ equation reduces to equations (1.24) and (1.27) for harmonics $h_{22 \mu}(r)$ when $\mu \neq 0$ and for harmonics $h_{m n 0}^{\mathrm{NN}}(r)$ when $\mu=0$. The colloid-nematic correlation functions $h^{\mathrm{CN}}(12)$ and $C^{\mathrm{CN}}(12)$ can also be written as a spherical harmonic expansion

$$
\begin{aligned}
f^{\mathrm{CN}}\left(\mathbf{r}_{12}, \omega_{2}\right)= & f_{000}^{\mathrm{CN}}\left(r_{12}\right)+f_{200}^{\mathrm{CN}}\left(r_{12}\right) Y_{20}\left(\hat{r}_{12}\right)+f_{020}^{\mathrm{CN}}\left(r_{12}\right) Y_{20}\left(\omega_{2}\right) \\
& +\sum_{|\mu| \leqslant 2} f_{22 \mu}^{\mathrm{CN}}\left(r_{12}\right) Y_{2 \mu}\left(\hat{r}_{12}\right) Y_{2 \mu}\left(\omega_{2}\right) .
\end{aligned}
$$

The axial symmetry of the bulk nematic allows equation (3.7) to be factorized into equations with different $\mu$. These equations can be Fourier transformed to obtain $k$-space equations in terms of Hankel transforms of harmonics of the correlation functions

$$
f_{m n \mu}^{\mathrm{CN}}(k)=4 \pi i^{m} \int_{0}^{\infty} r^{2} \mathrm{~d} r j_{m}(k r) f_{m n \mu}^{\mathrm{CN}}(r),
$$


where $j_{m}(x)$ is a spherical Bessel function. After Fourier transformation equation (3.7) for spherical harmonic coefficients can be written in the following form for $\mu=0$

$$
\hat{H}^{\mathrm{CN}}(k)\left[1-\hat{\rho} \hat{C}^{\mathrm{NN}}(k)\right]=\hat{C}^{\mathrm{CN}}(k),
$$

where the hat denotes matrices with elements

$$
\begin{aligned}
& C_{m n}^{\mathrm{CN}}(k)=4 \pi i^{m} \int_{0}^{\infty} r^{2} \mathrm{~d} r j_{m}(k r) C_{m n, 0}^{\mathrm{CN}}(r), \\
& H_{m n}^{\mathrm{CN}}(k)=4 \pi i^{m} \int_{0}^{\infty} r^{2} \mathrm{~d} r j_{m}(k r) h_{m n, 0}^{\mathrm{CN}}(r) .
\end{aligned}
$$

The matrix $\hat{C}^{\mathrm{NN}}(k)$ can be expressed in terms of the bulk Baxter functions (2.21)

$$
1-\hat{\rho}^{\mathrm{N}} \hat{C}^{\mathrm{NN}}(k)=\left[1-\hat{S}^{\mathrm{N}} \hat{Q}(-\mathrm{i} k)\right]\left[1-\hat{S}^{\mathrm{N}} \hat{Q}^{T}(\mathrm{i} k)\right] .
$$

One can then immediately solve equation (3.27) for the Hankel transforms of harmonics $h_{m n, 0}^{\mathrm{CN}}(r)$.

Similarly for $\mu \neq 0$

$$
h_{22 \mu}^{\mathrm{CN}}(k)\left[1-\rho\left\langle\left|Y_{2 \mu}\right|^{2}\right\rangle_{\omega} C_{22 \mu}^{\mathrm{NN}}\right]=C_{22 \mu}^{\mathrm{CN}}(k)
$$

and in terms of Laplace transforms

$$
h_{22 \mu}^{\mathrm{CN}}(k)\left[1-Q_{22 \mu}(-\mathrm{i} k)\right]\left[1-Q_{22 \mu}(\mathrm{i} k)\right]=C_{22 \mu}^{\mathrm{CN}}(K) .
$$

Finally, using the inverse Hankel transformation

$$
h_{m n \mu}^{\mathrm{CN}}(r)=4 \pi \frac{(-\mathrm{i})^{m}}{(2 \pi)^{3}} \int_{0}^{\infty} k^{2} \mathrm{~d} k j_{m}(k r) h_{m n \mu}^{\mathrm{CN}}(k)
$$

the total pair correlation function can be found in the $r$-space

$$
h^{\mathrm{CN}}\left(\mathbf{r}_{12}, \omega_{2}\right)=\sum_{m n} h_{m n 0}^{\mathrm{CN}}\left(r_{12}\right) Y_{m 0}\left(\hat{r}_{12}\right) Y_{n 0}\left(\omega_{2}\right)+\sum_{\mu \neq 0} h_{22 \mu}^{\mathrm{CN}}\left(r_{12}\right) Y_{2 \mu}\left(\hat{r}_{12}\right) Y_{2 \mu}^{*}\left(\omega_{2}\right) .
$$

In this numerical calculation for $C^{\mathrm{CN}}\left(\mathbf{r}_{12}, \omega_{2}\right)$ the ansatz (3.24) was used.

The non-direct part of the potential of the mean force for a pair of colloidal particles can be written in the form

$$
-\beta w^{\mathrm{CC}}(12)=h_{\mathrm{CC}}(12)-C_{\mathrm{CC}}(12)=-\sum_{l=0,2,4} \beta w_{l}^{\mathrm{CC}}\left(r_{12}\right) Y_{l 0}\left(\hat{r}_{12}\right) .
$$

After spherical harmonic expansions of the correlation functions using Fourier transforms of the coefficients of correlation functions in accordance with (3.8) we can write

$$
\begin{aligned}
-\beta w^{\mathrm{CC}}(k) & =\rho \sum_{m n} \sum_{n^{\prime} m^{\prime}} \sum_{\mu} C_{m n \mu}^{\mathrm{CN}}(k) h_{n^{\prime} m^{\prime} \mu}^{\mathrm{NC}}(k)\left\langle Y_{n \mu}(\omega) Y_{n^{\prime} \mu}^{*}(\omega)\right\rangle Y_{m \mu}(\hat{k}) Y_{m^{\prime} \mu}^{*}(\hat{k}) \\
& =\rho \sum_{m n} \sum_{\mu}\left[h_{m n^{\prime} \mu}^{\mathrm{CC}}(k)-C_{m n^{\prime} \mu}(k)\right] Y_{m \mu}(\hat{k}) Y_{m^{\prime} \mu}^{*}(\hat{k}),
\end{aligned}
$$

where indexes $m, m^{\prime}, n, n^{\prime}$ are equal to 0 or 2 .

Note that [38]

$$
Y_{m \mu}(\hat{k}) Y_{m^{\prime} \mu}(\hat{k})=\sum_{l}\left[\frac{(2 m+1)\left(2 m^{\prime}+1\right)}{(2 l+1)}\right]^{\frac{1}{2}}\left(\begin{array}{ccc}
m & m^{\prime} & l \\
\mu & -\mu & 0
\end{array}\right)\left(\begin{array}{ccc}
m & m^{\prime} & l \\
0 & 0 & 0
\end{array}\right) Y_{l 0}(\hat{k})
$$




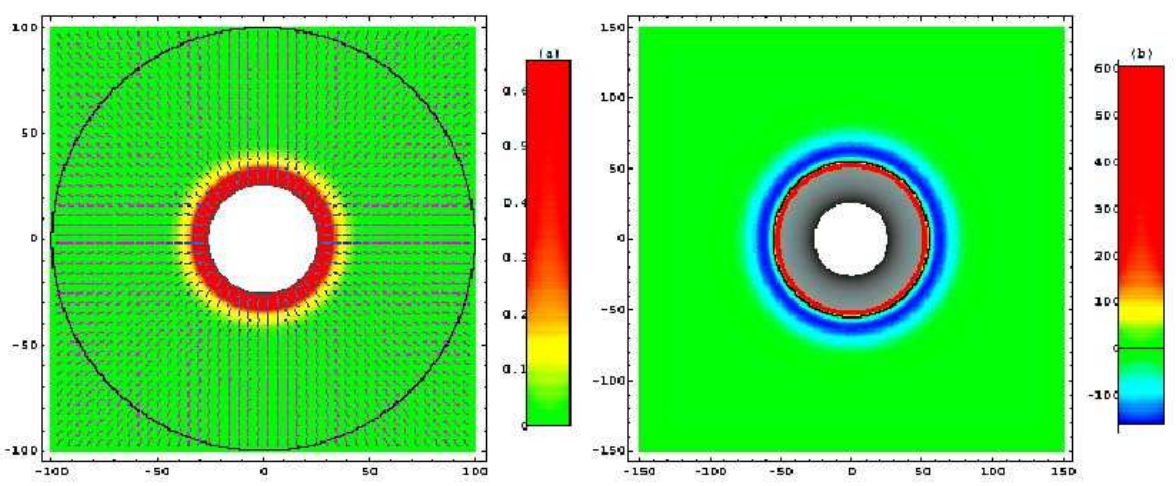

Figure 9. (a) Maps of the director $d_{m}(\mathbf{r})$ and the local ordering $S_{\mathrm{N}}(\mathbf{r})$ in the isotropic regime $(\eta=0.2)$ and zero external field $\beta W_{2}=0$. The colloidal particle is shown as a white circle of radius $\frac{1}{2} R=25$. The axes denote the distance from the center of colloidal particle in units of $\sigma$. The director field is indicated by bars showing the director orientation. The local ordering $\frac{1}{\rho} S_{\mathrm{N}}(\mathbf{r})$ is shown by color, red regions are more ordered. Positions where $\frac{1}{\rho} S_{\mathrm{N}}(\mathbf{r})$ equals the bulk order parameter $S_{2}$ are shown by thin black lines. (b) The potential of mean force $\beta w_{\mathrm{CC}}(12)$ at $\eta=0.2$ and $\beta W_{2}=0$. One colloidal particle is shown as a white circle of radius $\frac{1}{2} R$ and the grey stripe of width $\frac{1}{2} R$ surrounding it denotes the region inaccessible to the center of the other colloidal particle due to the hard-core repulsion. The center-center distance in units of $\sigma$ are indicated on both axes, and the color code is shown on the right. The blue regions are most attractive. The positions where the potential changes sign $\beta w_{\mathrm{CC}}(12)$ are shown by solid black lines.

and expression (3.36) can be rewritten in the form (3.35). In (3.37) $l$ changes from 0 to $m+$

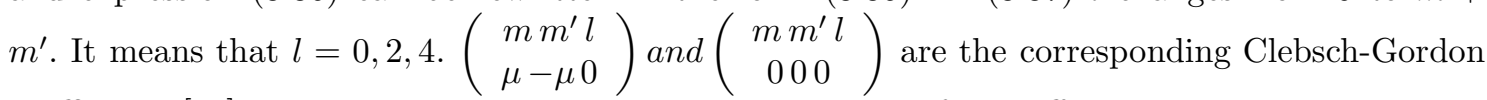
coefficients [38]. Due to the axial symmetry the contributions from different $\mu$ are separated again.

Some results of such calculations taken from [30, 33] are presented in figures 9-12 for perpendicular anchoring $\left(A_{\mathrm{C}}>0\right)$. These illustrate the assorted structure and interactions that can occur in nematic colloids under different conditions. Pictures labelled (a) are maps of the local ordering and director field around single colloidal particles. Note that the local ordering in the bulk is $\rho S_{2}$, where $\rho$ and $S_{2}$ are the bulk density and the order parameter. Pictures labelled (b) present the resulting potentials of mean force between colloidal pairs. Equilibrium configurations of two colloidal particles are defined by absolute minima of the potential of mean force shown with blue. We plot the results for two densities $\eta=\frac{1}{6} \pi \rho \sigma^{3}$, which describe two different regimes. $\eta=0.2$ corresponds to an isotropic phase at zero external field, whereas at $\eta=0.35$ the fluid is a stable nematic. One can see that in the isotropic regime the external field promotes the chain formation of colloids along its direction (figure 10). In the nematic regime tilted chains of colloidal particles (figure 11) can be transformed by increasing the external field, which promotes colloidal aggregation in the phase perpendicular to the field direction (figure 12). In sum, a rich variety of equilibrium colloidal structures can be promoted by different fields without changing the composition of the system.

Finally we consider the long-range behavior of colloid-colloid interactions [34]. These interactions result from colloid-induced distortions of nematic order and have been mainly described in the framework of phenomenological elastic theories [51, 52] which address the director distribution around a single colloidal particle. In the integral equation theory in MSA approximation, the asymptotes connected with elastic behavior are determined by the OZ-relations among harmonics with $\mu= \pm 1$. In the $k$-space these are 

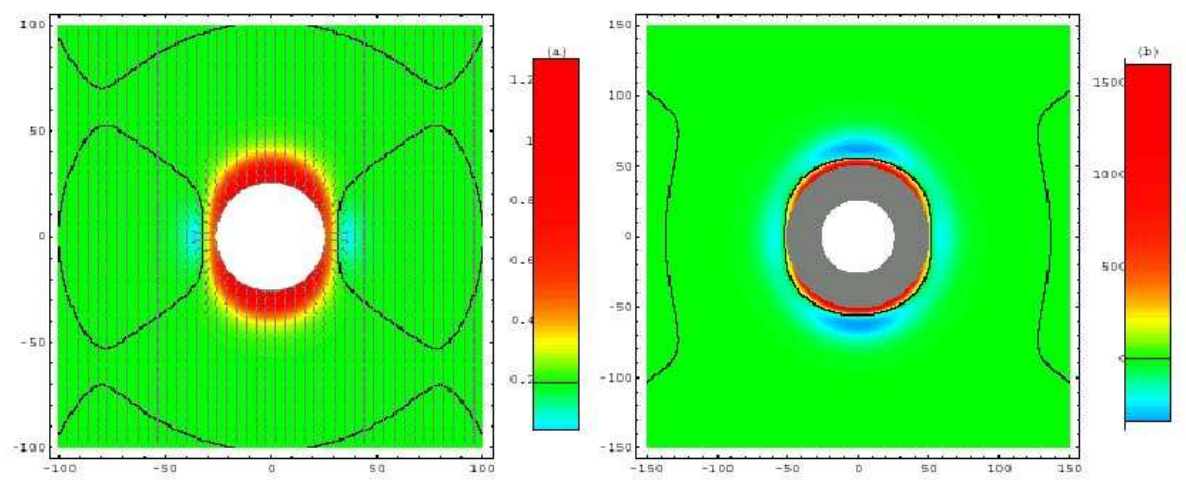

Figure 10. As in figure 9, but at non-zero external field $\beta W_{2}=0.1$. The field is directed along the vertical axis.
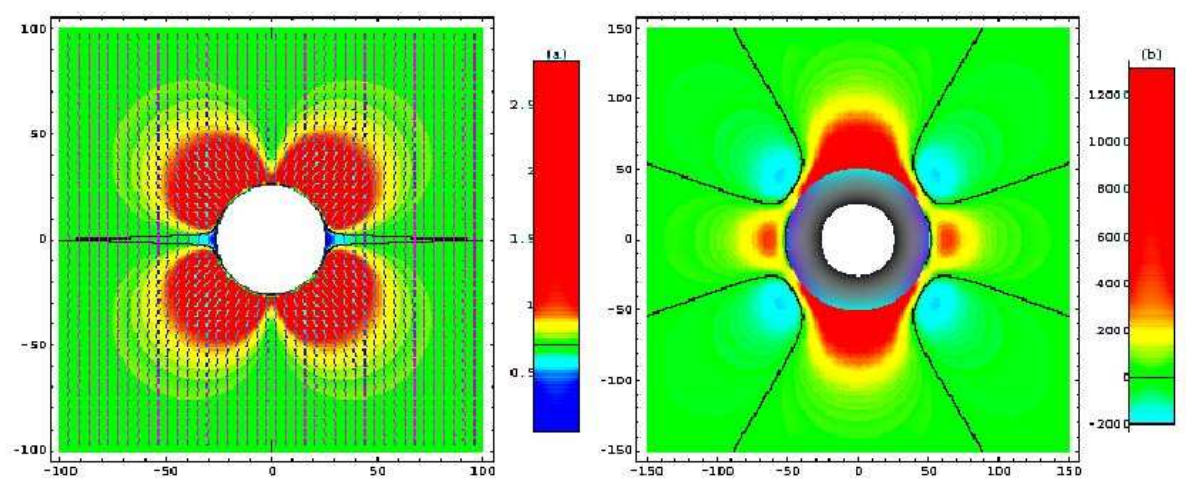

Figure 11. As in figure 9, but in the nematic region, $\eta=0.35$.
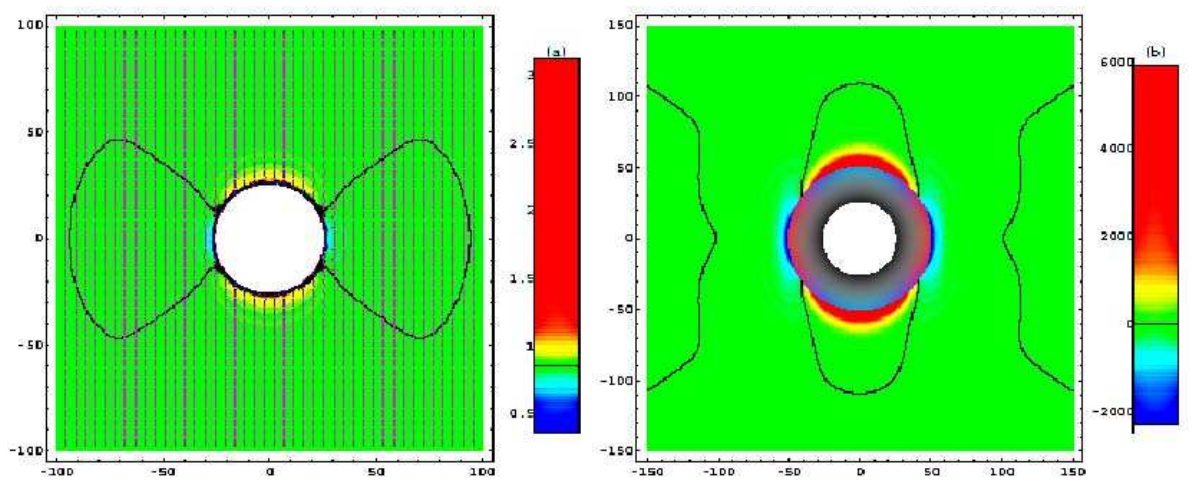

Figure 12. As in figure 11, but at non-zero external field $\beta W_{2}=1$. The field is directed along the vertical axis.

$$
\begin{aligned}
h_{221}^{\mathrm{NN}}(k) & =C_{221}^{\mathrm{NN}}(k)+C_{221}^{\mathrm{NN}}(k) \rho\left\langle\left|Y_{21}(\omega)\right|^{2}\right\rangle_{\omega} h_{221}^{\mathrm{NN}}(k), \\
h_{221}^{\mathrm{CN}}(k) & =C_{221}^{\mathrm{CN}}(k)+C_{221}^{\mathrm{CN}}(k) \rho\left\langle\left|Y_{21}(\omega)\right|^{2}\right\rangle_{\omega} h_{221}^{\mathrm{NN}}(k) \\
& =C_{221}^{\mathrm{CN}}(k)+h_{221}^{\mathrm{CN}}(k) \rho\left\langle\left|Y_{21}(\omega)\right|^{2}\right\rangle_{\omega} C_{221}^{\mathrm{NN}}(k), \\
h_{221}^{\mathrm{CC}}(k)-C_{221}^{\mathrm{CC}}(k) & =C_{221}^{\mathrm{CN}}(k) \rho\left\langle\left|Y_{21}(\omega)\right|^{2}\right\rangle_{\omega} h_{221}^{\mathrm{NN}}(k) .
\end{aligned}
$$


The equation (3.38) gives

$$
h_{221}^{\mathrm{NN}}(k)=\frac{C_{221}^{\mathrm{NN}}(k)}{\left[1-\rho\left\langle\left|Y_{21}(\omega)\right|^{2}\right\rangle_{\omega} C_{221}^{\mathrm{NN}}(k)\right]} .
$$

In the limit $k \rightarrow 0$ in accordance with (1.43)

$$
1-\rho\left\langle\left|Y_{21}(\omega)\right|^{2}\right\rangle_{\omega} C_{221}(k)=\frac{\beta W_{2}}{B_{2}}+k^{2} B^{2}+O\left(k^{4}\right),
$$

where

$$
B^{2}=\frac{\left\langle\left|Y_{21}(\omega)\right|^{2}\right\rangle_{\omega} \beta K}{\left[15 \rho S_{2}^{2}\right]},
$$

the elastic constant $K$ is given by (2.37). Now if we put (3.43) into (3.42), after the inverse zerothorder Hankel transformation, we will have

$$
h_{221}^{\mathrm{NN}}(r) \stackrel{r \rightarrow \infty}{\longrightarrow} C \frac{\exp (-r / \xi)}{r}
$$

where the decay length

$$
\xi=\left[K /\left(W_{2} \rho S_{2} 3 \sqrt{5}\right)\right]^{1 / 2}
$$

and the prefactor

$$
C=\left[4 \pi \rho B^{2}\left\langle\left|Y_{21}(\omega)\right|^{2}\right\rangle_{\omega}\right]^{-1}=\frac{3 B_{2}^{2}}{4 \pi \beta K} .
$$

In zero-field limit $W_{2}=0, \xi \rightarrow \infty$ and the result (3.45) coincides with our result (2.32) from the previous section.

For a sufficiently large spherical colloidal particle, the ansatz (3.24) was suggested. Noting that $j_{2}(x)=\frac{x^{2}}{15}\left(1-\frac{x^{2}}{14}+\cdots\right)$ at zero field

$$
C_{221}^{\mathrm{CN}}(k) \stackrel{k \rightarrow 0}{\longrightarrow}-4 \pi \frac{h_{221}^{\mathrm{WN}}\left(s=\frac{1}{2} \sigma\right)}{30 z_{c}} B R^{3} k^{2}+O\left(k^{4}\right)
$$

where $h^{\mathrm{WN}}\left(s=\frac{1}{2} \sigma\right)$ is the contact value of $h_{221}^{\mathrm{WN}}(s)$. From (3.34)

$$
h_{221}^{\mathrm{CN}}(k)=C_{221}^{\mathrm{CN}}(k)\left[1-\rho\left\langle\left|Y_{21}(\omega)\right|^{2}\right\rangle_{\omega} C_{221}^{\mathrm{NN}}(k)\right]^{-1} .
$$

Now using (3.48) and (3.43) in the limit $k \rightarrow 0$ we have

$$
h_{221}^{\mathrm{CN}}(k)=-\frac{4 \pi}{30} \frac{h_{221}^{\mathrm{WN}}\left(s=\frac{1}{2} \sigma\right)}{B z_{\mathrm{C}}} R^{3}+O\left(k^{2}\right) .
$$

Inverting the Hankel transform

$$
\frac{4 \pi}{(2 \pi)^{3}} i^{2} \int_{0}^{\infty} k^{2} \mathrm{~d} k j_{2}(k r)=-\frac{3}{4 \pi} \frac{1}{r^{3}}
$$

one finds

$$
h_{\mathrm{CN}}(12) \stackrel{r \rightarrow \infty}{\longrightarrow} \frac{1}{10} \frac{h_{221}^{\mathrm{WN}}\left(s=\frac{1}{2} \sigma\right)}{B z_{\mathrm{C}}} \frac{R^{3}}{r_{12}^{3}}\left[Y_{21}\left(\hat{r}_{12}\right) Y_{21}^{*}\left(\omega_{2}\right)+\text { c.c. }\right]
$$

where c.c. denotes the complex conjugate of the first term within the square brackets.

For a pair of colloidal particles labelled by subscripts $C$ and $C^{\prime}$ from equations (3.40) and (3.41) we have

$$
h_{221}^{\mathrm{CC}^{\prime}}(k)-C_{221}^{\mathrm{CC}^{\prime}}(k)=\frac{C_{221}^{\mathrm{CN}}(k) \rho\left\langle\left|Y_{21}(\omega)\right|^{2}\right\rangle_{\omega} C_{221}^{\mathrm{NC}^{\prime}}(k)}{1-\rho\left\langle\left|Y_{21}(\omega)\right|^{2}\right\rangle_{\omega} C_{221}^{\mathrm{NN}}(k)} .
$$


At zero field and small $k$ equation (3.53) takes the form

$$
h_{221}^{\mathrm{CC}^{\prime}}(k)-C_{221}^{\mathrm{CC}^{\prime}}(k) \rightarrow \rho\left\langle\left|Y_{21}(\omega)\right|^{2}\right\rangle_{\omega}(4 \pi)^{2} \frac{1}{z_{\mathrm{C}}} h_{221}^{\mathrm{WN}}\left(s=\frac{1}{2} \sigma\right) \frac{1}{z_{\mathrm{C}}} h_{221}^{W^{\prime} N}\left(s=\frac{1}{2} \sigma\right) \frac{R^{3} R^{\prime 3} k^{2}}{30^{2}} .
$$

The contribution of $\mu= \pm 1$ terms to the Fourier transforms of colloid-colloid potential of mean force is

$$
\begin{aligned}
{\left[h_{221}^{\mathrm{CC}^{\prime}}(k)-C_{221}^{\mathrm{CC}^{\prime}}(k)\right]\left[Y_{21}(\hat{k}) Y_{21}^{*}(k)+c . c .\right] } & =\left[h_{221}^{\mathrm{CC}^{\prime}}(k)-C_{221}^{\mathrm{CC}^{\prime}}(k)\right] 2\left[1+\frac{1}{7} \sqrt{5} Y_{20}(\hat{k})-\frac{4}{7} Y_{40}(\hat{k})\right] \\
& =\beta \sum_{l=0,2,4} w_{l}^{\mathrm{CC}^{\prime}}(k) Y_{l 0}(\hat{k}) .
\end{aligned}
$$

Although in $k$-space three terms occur on the right-hand side of equation (3.55), at zero field the $Y_{40}(\hat{k})$ term alone determines the asymptotic behavior of the potential of mean force in $r$-space. Using the inverse Hankel transformation for $l=4$ and noting that $k^{2} Y_{40}(\hat{k})$ becomes $105 Y_{40}(\hat{r}) /\left(4 \pi r^{5}\right)$ in $r$-space we obtain

$$
\beta w_{\mathrm{CC}^{\prime}}(r) \stackrel{r \rightarrow \infty}{\longrightarrow} \frac{8 \pi}{15} \frac{h_{221}^{\mathrm{WN}}\left(s=\frac{1}{2} \sigma\right)}{z_{\mathrm{C}}} \frac{h_{221}^{W^{\prime} N}\left(s=\frac{1}{2} \sigma\right)}{z_{C^{\prime}}} \rho\left\langle\left|Y_{21}(\omega)\right|^{2}\right\rangle_{\omega} \frac{R^{3} R^{\prime 3}}{r^{5}} Y_{40}(\hat{r}) .
$$

This result was obtained taking into account only the "elastic harmonics" $(\mu= \pm 1)$ in expansion (3.36). It is assumed that elastic deformations of the director field are dominant at long distances. However, this assumption becomes unsatisfactory near phase boundaries where fluctuations in local ordering are large.

These are results for the case when external field is absent and $\xi \rightarrow \infty$. But the correlation length also influences the orientational behavior of the effective colloid-colloid interaction. The so-called quadrupole interaction (3.56) that determines the long-range behavior at infinite $\xi$ transforms into a superposition of screened "multipoles" when $\xi$ is finite [34]

$$
\begin{aligned}
-\beta w_{\mathrm{CC}^{\prime}}(r) & \stackrel{r \rightarrow \infty}{\longrightarrow} \frac{4 \pi}{\xi^{5}} C\left(R, z_{\mathrm{C}}\right) C\left(R^{\prime}, z_{C^{\prime}}\right) \rho\left\langle\left|Y_{21}(\omega)\right|^{2}\right\rangle_{\omega} \\
& \times\left[-2 K_{0}\left(\frac{r}{\xi}\right)-\frac{10}{7} K_{2}\left(\frac{r}{\xi}\right) P_{2}(\hat{r})+\frac{24}{7} K_{4}\left(\frac{r}{\xi}\right) P_{4}(\hat{r})\right]
\end{aligned}
$$

where

$$
\begin{aligned}
& C\left(R, z_{\mathrm{C}}\right)=\frac{h_{221}^{\mathrm{WN}}\left(s=\frac{1}{2} \sigma\right)}{30 z_{\mathrm{C}}}\left[\frac{R^{4}}{8 \xi}+R^{3}\right] \\
& K_{0}(x)=\frac{\mathrm{e}^{-x}}{x}, \quad K_{2}(x)=\frac{1}{x^{3}}\left(3+3 x+x^{2}\right) \mathrm{e}^{-x} \\
& K_{4}(x)=\frac{1}{x^{5}}\left(105+105 x+45 x^{2}+10 x^{3}+x^{4}\right) \mathrm{e}^{-x}
\end{aligned}
$$

In the latest publication of T. Sokolovska [35] the problem of wall-colloid interaction in nematic solvents was discussed for "quadrupole" colloids. At weak field this interaction was obtained in the following form

$$
\begin{aligned}
-\beta w^{\mathrm{WC}}(\xi) & =\frac{\pi}{2} \rho\left\langle\left|Y_{21}(\omega)\right|^{2}\right\rangle_{\omega} \frac{h_{221}^{\mathrm{WN}}\left(s=\frac{1}{2} \sigma\right) h_{221}^{\mathrm{CN}}\left(s=\frac{1}{2} \sigma\right)}{z_{W} z_{\mathrm{C}}} \\
& \times \exp \left[-\frac{1}{\xi}\left(s-\frac{1}{2} \sigma\right)\right] \sin ^{2}\left(2 \vartheta_{s}\right) \frac{1}{\xi^{2}}\left[\frac{R^{4}}{8 \xi}+R^{3}\right] .
\end{aligned}
$$

This is a new type of an effective force acting on colloidal particles in the presence of an external field. In contrast to the so-called "image" interaction [53] that is always repulsive at long distances, the force identified in [35] can be attractive or repulsive, depending on the type of anchoring at the wall and colloidal surface $\left(A_{2}^{\mathrm{W}}, A_{2}^{\mathrm{C}}\right)$. The effective force on a colloidal particle decreases with the distance $s$ from the wall as $\exp (-s / \xi)$. 


\section{Conclusions}

The generalization and application of modern liquid state theory to the nematic and other liquid crystalline systems opens up new possibilities for the development of microscopic theory of liquid crystals. The leading role in this theory is played by the pair and singlet distribution functions, the knowledge of which makes it possible to describe the structure, thermodynamics, phase behavior, elastic and other properties depending on the nature of intermolecular interaction. A traditional way of calculating the pair distribution function is connected with the development of the integral equation theory which usually reduces to the solution of OZ equation with a corresponding closure relation.

In this paper we present the review of the integral equation theory for orientationally ordered fluids. The considered approach is based on self-consistent solution of OZ equation for the pair distribution function together with the TZLMBW equation for the singlet distribution function. It is shown that such an approach correctly describes the behavior of correlation functions of anisotropic fluids connected with the presence of Goldstone modes in the ordered phase in the zero-field limit. Due to this peculiarity in the orientationally-ordered state, the harmonics of the pair distribution function connected with correlations of the director transverse fluctuations become long-range ones in the zero-field limit. It is important to note that these harmonics do not give a direct contribution into the structure factor of nematic fluids. This phenomenon ensures the finite value of the structure factor in the limit of zero wave vector. The presence of Goldstone modes in an ordered phase is responsible for some specific properties of anisotropic fluids such as its elastic properties, multipole-like long-range asymptotes for effective interaction between colloids solved in nematic fluids and so on.

The capabilities of the formulated approach are illustrated through analytical results obtained in the framework of the mean spherical approximation for the Maier-Saupe nematogenic model. Out of the equation of state we select three types of phase diagrams depending on the ratio between isotropic and anisotropic interactions. For a strong isotropic attraction, we have the following phase transition between translational homogeneous phases: isotropic gas - isotropic liquid, isotropic gas - nematic and isotropic liquid - nematic. For a strong anisotropic interaction we observed a phase transition only between phases with different symmetries. In the isotropic repulsion case we also observed the nematic gas - nematic liquid phase transition. Using the Hansen-Verlet criterion [45] for crystallization, the point of coexistence of isotropic, nematic and crystalline phases was found. The effect of the disorienting field can significantly increase the region of the ordered fluid [23, 24].

The integral equation approach was also extended to a description of nematic fluid near a planar wall and a colloidal surface, as well as to colloidal-colloidal interaction in the presence of a uniform orienting field. The function $\rho_{\mathrm{NC}}\left(\omega, \mathbf{r}_{12}\right)=\rho f(\omega)\left[1+h_{\mathrm{NC}}\left(\omega, \mathbf{r}_{12}=\mathbf{r}_{1}-\mathbf{r}_{2}\right)\right]$ provides the distribution of nematic fluid about the colloidal particle. This function takes into account all the changes at a given point $\mathbf{r}_{\mathbf{1}}$ induced by the colloidal particle at $\mathbf{r}_{\mathbf{2}}$. They include the changes in the local density and in the orientational distribution of the nematic fluid. The function $\rho_{\mathrm{NC}}(\omega, \mathbf{r})$ defines the density-orientational profile of the generalized order parameter

$$
S_{\mathrm{C}}(\mathbf{r}, \hat{d})=\int P_{2}(\boldsymbol{\omega} \mathbf{d}) \rho_{\mathrm{NC}}(\omega, r) \mathrm{d} \omega
$$

which is connected with the director field configuration around the colloid $\hat{d}_{m}$ that maximizes $S_{\mathrm{C}}(\mathbf{r}, \mathbf{d})$ at a given point $\mathbf{r}$.

The application of anisotropic integral equation theory opens up new possibilities for the description of intercolloidal interactions in nematic solvents. Contrary to elastic theories [51, 52] which describe intercolloidal interactions only for asymptotically large distances, when correlation lengths are much larger than the particle size, the integral equation theory can describe the intercolloidal interactions at small and intermediate distances in the presence of an external field. These interactions are important for the description of colloidal phase diagrams and structure as well as other colloidal properties in order to be controlled with external fields. In contrast to phenomenological elastic theories, the integral equation method does not assume boundary conditions at colloidal surfaces but instead calculates them. From investigations of potentials of the mean force 
for pairs of identical colloidal particles with perpendicular anchoring [33] it was concluded that effective colloid-colloid interactions are determined by three main factors, namely the phase transition in confined geometry, depletion effects and elastic interactions between the nematic coating surrounding the colloidal particles. Varying the external field shifts the relative importance of these factors and significantly alters the effective interactions. In the framework of the integral equation theory it is also possible to involve colloidal particles of different size and form, ranging up to wall-colloid interactions. Effective potentials for colloidal pairs with asymmetric anchoring (e. g. perpendicular and parallel) are of interest as well. This can be also attributed to the effect of the presence of a third species in nematic colloids. A small amount of the second solvent (e. g., alkane impurities) can play a crucial role in opening the biphasic regions, and the consequent colloidal network formation [54].

In this paper we restrict ourselves to the consideration of the Maier-Saupe nematogenic model. The considered approach can be used for other anisotropic fluids. In [55, 56] this approach was used in the theory of magnetic fluids. This method can be also applied to several other interesting cases, such as the nematic phase of hard convex bodies, as well as dipolar and ferro-fluids.

\section{Acknowledgement}

The author thanks A. Trokhymchuk for the invitation to prepare this review.

\section{References}

1. Yukhnovsky I.R., Holovko M.F., The Statistical Theory of Classical Equilibrium Systems. Naukova Dumka, Kyiv, 1980.

2. Hansen J.P., McDonald I.R., Theory of Simple Liquids.Academic Press, Oxford, 2004.

3. Henderson D. - In: Fundamentals of inhomogeneous fluids, ed. by D. Henderson, M. Dekker. New York, 1992.

4. Bogolubov N.N., Problems of dynamical theory in statistical physics.Gostekhizdat, Moscow, 1946. Selected papers, vol.2, p. 99-196, Naukova Dumka, Kyiv, 1970.

5. Triezenberg D.G., Zwanzig R., Phys. Rev. Lett., 28, 1183, 1972.

6. Lovett R., Mou C.Y., Buff F.P., J. Chem. Phys., 65, 370, 1976.

7. Wertheim M., J. Chem. Phys., 65, 2377, 1976.

8. Bogolubov N.N., Selected papers, vol. 3, p.174, Naukova Dumka, Kyiv, 1971.

9. Baus M., Colot L.J., Wu X.G., Xu H., Phys. Rev. Lett. 59, 2184, 1987.

10. Velasko E., Somoza A.M., Mederos L., J. Chem. Phys. 102, 8107, 1995.

11. Lipszyc K., Kloczkowski A., Acta Physica Polonica, A63, 805, 1983.

12. Zhong H., Petschek R., Phys. Rev. E51, 2263, 1995.

13. Zhong H., Petschek R., Phys. Rev. E53, 4944, 1996.

14. Holovko M.F., Sokolovska T.G., J. Mol. Liq. 82, 161, 1999.

15. Stelzer L., Longa L., Trebin H.R., J. Chem. Phys. 103, 3098, 1995.

16. Lomba E., Martin C., Almazza N.G., Lado F., Phys. Rev. E74, 021503, 2006.

17. Sokolovska T.G., Holovko M.F., Cond. Matter Phys. 109, No 11, 1997.

18. de Gennes P.G., The physics of liquid crystals. Oxford university press, 1974.

19. Holovko M.F., Sokolovska T.G., Ukr. Phys. J., 41, 933, 1996.

20. Sokolovska T.G., Holovko M.F., Sokolovskii P.O., Ukr. Phys. J., 42, 1304, 1997.

21. Perera A., Phys. Rev. E60, 2912, 1999.

22. Mishra P., Singh S.L., Ram J., Singh Y., J. Chem. Phys, 127, 044905, 2007.

23. Sokolovska T.G., Sokolovskii R.O., Holovko M.F., Phys. Rev. E69, 6771, 2000.

24. Sokolovska T.G., Sokolovskii R.O., Holovko M.F., Phys. Rev. 64, 051710, 2001.

25. Lado F., Lomba E., Martin C., J. Mol. Liq. 112, 51, 2004.

26. Lomba E., Martin C., Almazza N.G., Lado F., Phys. Rev. E71, 046132, 2005.

27. Henderson D., Abraham F.F., Barker J.A., Mol. Phys. 100, 129, 2002; 31, 1291, 1976.

28. Sokolovska T.G., Sokolovskii R.O., Patey G.N., Phys. Rev. Lett. 92, 185508, 2004.

29. Sokolovska T.G., Sokolovskii R.O., Patey G.N., J. Chem. Phys. 122, 034703, 2005.

30. Sokolovska T.G., Sokolovskii R.O., Patey G.N., J. Chem. Phys. 122, 124907, 2005.

31. Sokolovska T.G., Sokolovskii R.O., Patey G.N., J. Chem. Phys. 125, 034903, 2006. 
32. Sokolovska T.G., Sokolovskii R.O., Patey G.N., Cond. Matt. Phys. 10, 407, 2007.

33. Sokolovska T.G., Sokolovskii R.O., Patey G.N., Phys. Rev. E73, 020701(R), 2006.

34. Sokolovska T.G., Sokolovskii R.O., Patey G.N., Phys. Rev. E77, 041701, 2008.

35. Sokolovska T.G., Patey G.N., J. Phys.: Condens. Matter 21, 245105, 2009.

36. Maier W., Saupe A., Z. Naturforsch, A14, 882, 1954.

37. J.S.Rowlinson, B. Widom. Molecular Theory of Capillarity. Clarendon Press, Oxford, 1982.

38. Gray C.G., Gubbins K.E., Theory of molecular fluids. Clarendon press, Oxford, 1984.

39. Blum L., Hoye J.S., J. Stat. Phys. 12, 317, 1978.

40. Kloczkowski A., Stecki J., Mol. Phys. 46, 13, 1982.

41. Kac M., Phys. Fluids. 2, 8, 1959.

42. Pastore G., Mol. Phys. 63, 731, 1988.

43. Sokolovska T.G., Dissertation. Lviv, Institute for Condensed Matter Physics, 1998.

44. Hoye J.S., Stell G., J. Chem. Phys. 67, 439, 1977.

45. Hansen J.P., Verlet L., Phys. Rev. 184, 150, 1969.

46. Poniewerski A., Stecki J., Phys. Rev. A25, 2368, 1982.

47. Frenkel D., In "Liquids, Freezing and Glass Transition" ed. by J.P. Hansen, D. Levesque and J. JinnJustine. Elsevier Science Publishers, British Vancouver, 1991.

48. Blum L., Stell G., J. Stat. Phys. 15, 439, 1976.

49. Perram J.W., Mol. Phys. 30, 1505, 1975.

50. Soviak E.M., Holovko M.F., Kravtsiv I.Y., Preprint icmp-10-03U, Lviv, 2010.

51. Lubensky T.C., Pettey D., Currier N., Stark H., Phys. Rev. E57, 610, 1998.

52. Pergamenshuk V., Uzunova V., Cond. Matter Phys. 13, 2010, (!pages from current issue).

53. Terentjev E.M., Phys. Rev. E51, 1330, 1995.

54. Vollmer D., Hinze G., Poon W.C.K., Cleaver J., Cates M.E., J. Phys.: Condens. Matter. 16, L227, 2004.

55. Sokolovska T.G., Physica A, 253, 459, 1997.

56. Sokolovska T.G., Sokolovskii R.O., Phys. Rev. E59, R3819, 1999.

\title{
Теорія інтегральних рівнянь для нематичних флюїдів
}

\author{
М.Ф.Головко \\ Інститут фізики конденсованих систем НАН України, вул. Свєнціцького, 1, Львів, 79011, Україна
}

Традиційний формалізм у теорії рідин, що базується на розрахунку парної функції розподілу, узагальнений на нематичні плини. Розглядуваний підхід базується на розв'язку орієнтаційнонеоднорідного рівняння Орнштейна-Церніке в поєднанні з рівнянням Трайцінберга-ЦванцігаЛовета-Моу-Бафа-Вертгайма. Показано, що даний підхід коректно описує поведінку кореляційних функцій анізотропних флюїдів, обумовлену наявністю голдстоунівських мод у впорядкованій фазі при відсутності упорядковуючого зовнішнього поля. Ми зосереджуємось на обговоренні аналітичних результатів отриманих у співпраці з Т.Г. Соколовською в рамках середньо-сферичного наближення для нематогенної моделі Майєра-Заупе. Представлена фазова діаграма цієї моделі. Встановлено, що в нематичному стані гармоніки парної кореляційної функції, пов'язані з кореляціями флуктуацій поперечних до напрямку директора, стають далекосяжними при відсутності впорядковуючого поля. Показано, що така поведінка функції розподілу нематичного флюїду приводить до дипольно- та квадрупольно-подібних далекосяжних асимптотик ефективної міжколоїдної взаємодії в нематичних флюїдах, передбаченої раніше феноменологічними теоріями.

Ключові слова: парна функція розподілу, теорія інтегральних рівнянь, нематогенна модель Майєра-Заупе, моди Голдстоуна, колоїдно-нематична суміш 
